\title{
A MICROSCOPIC, BUT NOT SELF-CONSISTENT APPROACH TO NUCLEAR BINDING AND DEFORMATION ENERGIES ${ }^{\dagger}$
}

\author{
C. M. KO ${ }^{\dagger \dagger}, \mathrm{H}$. C. PAULI ${ }^{\dagger \dagger}, \mathrm{M}$. BRACK $:$ and G. E. BROWN \\ Department of Physics, State University of New York at Stony Brook, New York 11790, USA
}

Received 27 August 1974

\begin{abstract}
Using various versions of the Skyrme force and Negele's interaction, we calculate deformation energies of nuclei by evaluating the expectation value of the many-body Hamiltonian in wave functions taken to be antisymmetrized products of singlc-particle functions. These single-particle functions are eigenfunctions of a phenomenological potential, here taken to be a deformed Woods-Saxon well. The method can be thought of as an extension of the Strutinsky shell-correction method, to make the connection with the two-body interaction. The method employed here is checked by comparison with Hartree-Fock (HF) results; our method is, however, much faster than the HF method, and, therefore, suitable for a wide range of problems where one tests the sensitivity of results to changes in the two-body interaction. A fairly good agreement with the HF method is obtained for ground-state energies, radii and deformations, as well as for deformations of shape isomers. The main discrepancy is that our energies tend to increase slightly too rapidly with deformation, indicating that we may not have chosen the best phenomenological well. Two-dimensional energy surfaces, which agree quite well with those from the Strutinsky method, are found for ${ }^{240} \mathrm{Pu}$.
\end{abstract}

\section{Introduction}

This work is motivated by the desire to relate deformation-energy surfaces of nuclei back to the effective nucleon-nucleon interaction in nuclei. More specifically, we take the expectation value of the many-body Hamiltonian, inclusive of two-body interactions, between antisymmetrized products of single-particle wave functions calculated in a phenomenological potential, here taken to be a deformed Woods-Saxon well ${ }^{1,2}$ ). We shall adduce arguments to show that this procedure gives a good approximation to the energy as function of deformation.

One already has the well-established Strutinsky shell-correction method for obtaining the multidimensional deformation-energy surfaces ${ }^{3-6}$ ). Our method can be viewed as an extension of the Strutinsky procedure; we wish to make the connection back to the nucleon-nucleon interaction. Because of the complexity in detail of the problem in the case of heavy nuclei, we are able to do this only with somewhat schematic two-body interactions such as the density-dependent zero-range interactions, of the type of the Skyrme ${ }^{7,8}$ ) and Negele forces ${ }^{9}$ ).

t Work supported in part by the US Atomic Energy Commission.

tt Present address: Department of Physics, McMaster University, Hamilton, Ontario, Canada L8S 4M1.

t+† Present address: Max-Planck-Institut für Kernphysik, P.O. Box 1248, D-69 Heidelberg 1, Germany.

\# Present address: Niels Bohr Institutet, Blegdamsvej 17, DK-2100 Kobenhavn- $\varnothing$. 
We can check our method against constrained HF calculations employing the same interactions ${ }^{8,10-12}$ ). The constrained HF method gives the path of lowest energy, for a given variation in constraint, in the multidimensional space. Up to the present time, only one constraint - essentially the quadrupole moment - has been able to be included in the HF calculations.

Our method has the advantage of being much faster and giving more information (in that it gives the entire multidimensional energy surface) than the HF calculations carried out to date. However, belief in the reliability of our method rests on the comparison with the presumably more accurate results of HF for the particular path found in the latter method. We find not only qualitatively, but even quantitatively, comparable results.

The HF calculations ${ }^{8,10-12}$ ) of the Orsay group (Brink, Flocard, Quentin, Vautherin and Veneroni) have enjoyed considerable success, and we do not propose our method as an alternative to theirs. But, when one wants to see what effects small changes in parameters, etc., will have, it is cumbersome to go through the entire selfconsistent calculation, and we claim that our method should be sufficiently precise for a rapid estimation of such effects.

It also seems to us that the HF approach is in some sense like a black box. The average one-body potential and the single-particle wave functions, generating each other self-consistently, are inseparably interwoven with the nucleon-nucleon interactions used. Although a determination of some of the liquid-drop parameters for a given force is possible ${ }^{10,13,14}$ ), the interplay of the detailed components of the force and the obtained properties of finite nuclei, especially e.g. the shell effects, are still very little understood. The self-consistent potentials which we see at the end of the calculation are very close to the phenomenological shell model potentials used so far. Therefore the question arises how important the exact self-consistency actually is and whether it is worth paying for it so much numerical computation time.

Our purpose is not so much to reproduce the experimental results at this stage of the development, but to compare the results to the HF results on one hand and those of Strutinsky calculations on the other hand, the latter ones being obtained with the same single-particle model. The quality of agreement with the self-consistent results will test the validity of the present approximation as a whole and tell us something about the importance of the self-consistency condition. The agreement with the Strutinsky calculations might give some insight into the origins of the shell structure and the role of the nuclear force.

It should be clear that the method can be gradually improved towards the HF method by minimizing the total energy with respect to a large number of parameters entering the average field, i.e. by explicitly performing some of the variations which are implicitly done in the HF approach. Although this would make the method more and more cumbersome and less useful for practical applications, such a procedure could help to shed some light on the mechanisms happening inside the HF "black box".

For practical application, however, we will take advantage of the relative simplicity 
of the method as compared to the HF approach, and try to use an average potential without optimizing it in each case.

\section{Presentation of the formalism}

The mathematical simplicity of the Skyrme-type effective interactions lies in the fact that the expectation value of the total Hamiltonian between Slater determinants of a set of orthonormalized single-particle wave functions $\psi_{i}(r)$, for a doubly even nucleus, can be written as a three-dimensional integral over an energy density $H(r)$,

$$
E=\langle T+V\rangle=\int \mathrm{d} \boldsymbol{r} H(\boldsymbol{r}),
$$

which is a functional of the nucleon density $\rho(\boldsymbol{r})$, the kinetic energy density $\tau(\boldsymbol{r})$ and some spin-orbit density $\boldsymbol{J}(\boldsymbol{r})$ defined in terms of the single-particle wave functions $\psi_{i}(r)$ and some occupation numbers $n_{i}$

$$
\begin{aligned}
\rho(\boldsymbol{r}) & =\sum_{i} n_{i}\left|\psi_{i}(\boldsymbol{r})\right|^{2}, \\
\tau(\boldsymbol{r}) & =\sum_{i} n_{i}\left|\nabla \psi_{i}(\boldsymbol{r})\right|^{2}, \\
\boldsymbol{J}(\boldsymbol{r}) & =-i \sum_{i} n_{i} \psi_{i}^{*}(\boldsymbol{r})(\boldsymbol{\nabla} \times \boldsymbol{\sigma}) \psi_{i}(\boldsymbol{r}),
\end{aligned}
$$

where the $n_{i}$ fulfil the equations

$$
\sum_{i} n_{i}=N \text { or } Z \text {. }
$$

These densities are defined separately for each kind of nucleon (neutrons, protons); we denote them with the indices $\mathbf{n}$ and $\mathbf{p}$ where it is necessary to avoid confusion (in these cases we define $\rho(\boldsymbol{r})=\rho_{\mathrm{n}}(\boldsymbol{r})+\rho_{\mathrm{p}}(\boldsymbol{r})$ etc.).

In the following we shall restrict the presentation to the case of the Skyrme interaction as used in the recent literature ${ }^{8,10-12}$ ). The formalism applies however in the same way to the modified delta interaction of Moszkowski ${ }^{15-18}$ ) or the interaction of Negele in the density matrix expansion (DME) ${ }^{9}$ ), an application of which is also given in the next section.

For the Skyrme force, the energy density in eq. (2.1) has the explicit form

$$
\begin{aligned}
H(\boldsymbol{r})= & \frac{\hbar^{2}}{2 m} \tau(\boldsymbol{r})+\frac{1}{2} \phi_{\mathrm{CD}}(\boldsymbol{r}) \rho_{\mathrm{p}}(\boldsymbol{r})+\frac{3}{4} \phi_{\mathrm{CE}}(\boldsymbol{r}) \rho_{\mathrm{p}}(\boldsymbol{r}) \\
& +\frac{1}{2} t_{0} \rho^{2}(\boldsymbol{r})-\frac{1}{2} t_{0}\left(\rho_{\mathrm{n}}^{2}(\boldsymbol{r})+\rho_{\mathrm{p}}^{2}(\boldsymbol{r})\right) \\
& +\frac{1}{4}\left(t_{1}+t_{2}\right) \rho(\boldsymbol{r}) \tau(\boldsymbol{r})+\frac{1}{8}\left(t_{2}-t_{1}\right)\left(\rho_{\mathrm{n}}(\boldsymbol{r}) \tau_{\mathrm{n}}(\boldsymbol{r})+\rho_{\mathrm{p}}(\boldsymbol{r}) \tau_{\mathrm{p}}(\boldsymbol{r})\right) \\
& +\frac{1}{16}\left(t_{2}-3 t_{1}\right) \rho(\boldsymbol{r}) \nabla^{2} \rho(\boldsymbol{r})+\frac{1}{32}\left(t_{2}+3 t_{1}\right)\left(\rho_{\mathrm{n}}(\boldsymbol{r}) \nabla^{2} \rho_{\mathrm{n}}(\boldsymbol{r})+\rho_{\mathrm{p}}(\boldsymbol{r}) \nabla^{2} \rho_{\mathrm{p}}(\boldsymbol{r})\right) \\
& +\frac{1}{4} t_{3} \rho(\boldsymbol{r}) \rho_{\mathrm{n}}(\boldsymbol{r}) \rho_{\mathrm{p}}(\boldsymbol{r}) \\
& +\frac{1}{4} x_{0} t_{0} \rho^{2}(\boldsymbol{r})-\frac{1}{2} x_{0} t_{0}\left(\rho_{\mathrm{n}}^{2}(\boldsymbol{r})+\rho_{\mathrm{p}}^{2}(\boldsymbol{r})\right) \\
& -\frac{1}{2} W_{0} \rho(\boldsymbol{r}) \nabla \cdot \boldsymbol{J}(\boldsymbol{r})-\frac{1}{2} W_{0}\left(\rho_{\mathrm{n}}(\boldsymbol{r}) \nabla \cdot \boldsymbol{J}_{\mathrm{n}}(\boldsymbol{r})+\rho_{\mathrm{p}}(\boldsymbol{r}) \nabla \cdot \boldsymbol{J}_{\mathrm{p}}(\boldsymbol{r})\right),
\end{aligned}
$$


with

$$
\begin{aligned}
& \phi_{\mathrm{CD}}(\boldsymbol{r})=e^{2} \int \frac{\mathrm{d} \boldsymbol{r}^{\prime} \rho_{p}\left(\boldsymbol{r}^{\prime}\right)}{\left|\boldsymbol{r}-\boldsymbol{r}^{\prime}\right|}, \\
& \phi_{\mathrm{CE}}(\boldsymbol{r})=-\left(\frac{3}{\pi}\right)^{\frac{1}{3}} e^{2} \rho_{\mathrm{p}}^{\frac{3}{3}}(\boldsymbol{r}) .
\end{aligned}
$$

The parameters $t_{0}, t_{1}, t_{2}, t_{3}, x_{0}$ and $W_{0}$ characterize the Skyrme force; different sets of them are given in the next section. In the Negele-DME interaction, some functionals of the densities $\rho_{\mathrm{n}}$ and $\rho_{\mathrm{p}}$ replace the constant parameters $t_{0}, t_{1}, t_{2}, t_{3}$ and $x_{0}$.

We shall discuss briefly, now, the constrained HF method ${ }^{11}$ ), which we use in order to get results to check our expectation-value method.

With the assumption of Slater determinants as total wave functions, the binding energy of a nucleus is given by eq. (2.1) and thus depends on the choice of the wave functions $\psi_{i}(\boldsymbol{r})$. The best set can be found by varying them independently until the total energy (2.1) is stationary. This variational procedure is normally performed with some subsidiary conditions (constraint) which guarantee a conserved number of particles (Lagrange multipliers $\mu_{\mathrm{n}}$ and $\mu_{\mathrm{p}}$ ) and a certain shape of the nucleus, e.g. a given quadrupole moment $Q_{0}=\langle Q\rangle$ (Lagrange multiplier $\lambda$ ),

$$
\delta\left[\int H(\boldsymbol{r}) \mathrm{d} \boldsymbol{r}-\mu_{\mathrm{n}} N-\mu_{\mathrm{p}} Z+f(\lambda,\langle Q\rangle)\right]=0 .
$$

The simplest constraint for the quadrupole moment is $f(\lambda,\langle Q\rangle)=\lambda\langle Q\rangle$; it has been shown, by Flocard et al. ${ }^{11,12}$ ), however, that at least a quadratic constraint is necessary to obtain all points of the deformation energy curves,

$$
f(\lambda,\langle Q\rangle)=\frac{1}{2} C(\lambda-\langle Q\rangle)^{2}
$$

The variational principle eq. (2.5), applied to the single-particle wave functions $\psi_{i}(r)$, leads to a set of HF equations which for the Skyrme energy density $(2.4)$ has the form $(q=\mathrm{n}, \mathrm{p})$

$$
\begin{aligned}
H_{q} \psi_{i}^{q}(\boldsymbol{r}) & =\left\{\frac{\hbar^{2}}{2 m} \nabla \cdot M_{q}(\boldsymbol{r}) \nabla+U_{q}(\boldsymbol{r})+\left[\nabla W_{q}(\boldsymbol{r}) \times(-i) \nabla\right] \cdot \sigma+\left.\frac{\partial f}{\partial\langle Q\rangle}\right|_{Q_{0}} \hat{q}(\boldsymbol{r})\right\} \psi_{i}^{q}(\boldsymbol{r}) \\
& =\varepsilon_{i}^{q} \psi_{i}^{q}(\boldsymbol{r}) .
\end{aligned}
$$

In eq. (2.7), $\hat{q}(\boldsymbol{r})$ is the quadrupole operator; the effective mass $M_{q}(\boldsymbol{r})$, the average potential $U_{q}(\boldsymbol{r})$ and the spin-orbit field $W_{q}(\boldsymbol{r})$ are functionals of the densities eq. (2.2) and their derivatives

$$
M_{q}(\boldsymbol{r})=1+\frac{m}{8 \hbar^{2}}\left[\left(t_{1}+t_{2}\right) \rho(\boldsymbol{r})+\frac{1}{2}\left(t_{2}-t_{1}\right) \rho_{q}(\boldsymbol{r})\right]
$$




$$
\begin{aligned}
U_{q}(\boldsymbol{r}) & =t_{0}\left[\left(1+\frac{1}{2} x_{0}\right) \rho(\boldsymbol{r})-\left(x_{0}+\frac{1}{2}\right) \rho_{q}(\boldsymbol{r})\right] \\
& +\frac{1}{4}\left(t_{1}+t_{2}\right) \tau(\boldsymbol{r})+\frac{1}{8}\left(t_{2}-t_{1}\right) \tau_{q}(\boldsymbol{r}) \\
& +\frac{1}{8}\left(t_{2}-3 t_{1}\right) \nabla^{2} \rho(\boldsymbol{r})+\frac{1}{16}\left(t_{2}+3 t_{1}\right) \nabla^{2} \rho_{q}(\boldsymbol{r}) \\
& +\frac{1}{4} t_{3}\left(\rho^{2}(\boldsymbol{r})-\rho_{q}^{2}(\boldsymbol{r})\right) \\
& -\frac{1}{2} W_{0}\left[\nabla \cdot \boldsymbol{J}(\boldsymbol{r})+\boldsymbol{\nabla} \cdot \boldsymbol{J}_{q}(\boldsymbol{r})\right] \\
& +\delta_{q, \mathrm{p}}\left[\phi_{\mathrm{CD}}(\boldsymbol{r})+\phi_{\mathrm{CE}}(\boldsymbol{r})\right], \\
& W_{q}(\boldsymbol{r})=\frac{1}{2} W_{0}\left[\rho(\boldsymbol{r})+\rho_{q}(\boldsymbol{r})\right] .
\end{aligned}
$$

In the equation for the average potential $U_{q}(r)$ we have included the Coulomb potential for the protons, i.e. $\phi_{\mathrm{CD}}$ and $\phi_{\mathrm{CE}}$, where the latter stands for the exchange potential calculated in the Slater approximation $\left.{ }^{9}\right)$.

The eqs. (2.2), (2.7) and (2.8) build a rather complicated system of equations which can be solved numerically by an iterative procedure. Our expectation-value method here consists in replacing eqs. (2.8) by the introduction of some phenomenological average fields, for which a fairly good understanding has been developed in the past. These fields shall have a certain depth $V$, a radius $R$, and a certain diffusivity $a$, i.e. we profitably take Fermi functions,

$$
\begin{gathered}
M_{q}(r) \leftrightarrow M_{q}(r)=M_{0}\left[1+\exp \left(\frac{r-R_{\mathrm{m}}}{a_{\mathrm{m}}}\right)\right]^{-1}+1, \\
U_{q}(\boldsymbol{r}) \leftrightarrow V_{q}(\boldsymbol{r})=V_{0}\left[1+\exp \left(\frac{r-R_{\mathrm{v}}}{a_{\mathrm{v}}}\right)\right]^{-1}+\delta_{q, \mathrm{p}} \phi_{\mathrm{C}}(\boldsymbol{r}), \\
W_{q}(\boldsymbol{r}) \leftrightarrow S_{q}(\boldsymbol{r})=\chi_{0}\left[1+\exp \left(\frac{r-R_{\mathrm{s}}}{a_{\mathrm{s}}}\right)\right]^{-1} .
\end{gathered}
$$

We may note already that the radii as well as the diffusivities may be different in the three fields, which may also differ for neutrons and protons; in the most general case we have thus 18 independent parameters. The way they have been chosen is discussed in the next section.

We use now these phenomenological potentials to construct a phenomenological single-particle Hamiltonian,

$$
\begin{aligned}
H_{\mathrm{ph}} \phi_{i}^{q}(\boldsymbol{r}) & =\left\{\frac{\hbar^{2}}{2 m} \nabla \cdot M_{q}(\boldsymbol{r}) \nabla+V_{q}(\boldsymbol{r})+\left[\nabla S_{q}(\boldsymbol{r}) \times(-i) \nabla\right] \cdot \sigma\right\} \phi_{i}^{q}(\boldsymbol{r}) \\
& =e_{i}^{q} \phi_{i}^{q}(\boldsymbol{r}),
\end{aligned}
$$

to generate new single-particle wave functions $\phi_{i}$, which we insert into eq. (2.2) in order to define the three relevant densities. Even though these phenomenological potentials may not be realistic in the sense that they deviate appreciable from the self- 
consistent potentials, they are useful to define reasonable trial wave functions in the sense of the variational theorem of Ritz.

The self-consistent and the phenomenological Schrödinger equation, which we have written down in eqs. (2.7) and (2.10), respectively, differ however in the important aspect, that the latter does not contain the so called external field

$$
\lambda \hat{q}(\boldsymbol{r}) \text { or }\left.\frac{\partial f}{\partial\langle Q\rangle}\right|_{Q_{0}} \hat{q}(\boldsymbol{v})
$$

It is claimed by Bassichis et al. ${ }^{19,20}$ ) that the phenomenological average field $V_{q}(r)$ contains what one could term an external field away from extrema of the energy. Thus, one should be careful in trying to interpret our phenomenological potential directly as a self-consistent field.

Removal energies $r_{i}$ are defined as

$$
r_{i}=\frac{\delta\langle H\rangle}{\delta n_{i}}=\int \psi_{i}^{*}(\boldsymbol{r}) \frac{\delta H(\boldsymbol{r})}{\delta \rho_{i}} \psi_{i}(\boldsymbol{r}) \mathrm{d} \boldsymbol{r} .
$$

These removal energies are related to the single-particle energies occurring in the HF equations by

$$
r_{i}=\varepsilon_{i}-\left.\frac{\partial f}{\partial\langle Q\rangle}\right|_{Q_{0}} q_{i}
$$

Thus, the removal energies $r_{i}$ differ from the eigenenergies $\varepsilon_{i}$ of the HF eq. (2.7) by the quantity $-\partial f /\left.\partial\langle Q\rangle\right|_{Q_{0}} q_{i}$, where $q_{i}$ is the quadrupole moment of the $i$ th level. For equilibrium shapes, where no constraint is needed, the two are of course identical.

In the phenomenological case, the situation is different, because in lowest-order approximation the removal energy is given by the eigenvalue

$$
r_{i} \approx e_{i}
$$

even for non-equilibrium deformations. Therefore, by a physical interpretation we identify the two, i.e.

$$
e_{i} \leftrightarrow \varepsilon_{i}-\left.\frac{\partial f}{\partial\langle Q\rangle}\right|_{Q_{0}} q_{i},
$$

and omit the constraint in the single-particle equation.

The removal energies, given by eq. (2.12) can be calculated with the wave function alone

$$
r_{i}=\int \mathrm{d} \boldsymbol{r} \psi_{i}^{+}\left\{\frac{\hbar^{2}}{2 m} \nabla \cdot M(\boldsymbol{r}) \nabla+U(\boldsymbol{r})+[\nabla W(\boldsymbol{r}) \times(-i) \nabla] \cdot \sigma\right\} \psi_{i}(\boldsymbol{r})
$$

Approximate values of $r_{i}$ can be obtained by replacing the self-consistent wave function $\psi_{i}$ by the phenomenological wave functions in the definition of the densities, eq. (2.2) and the equations for the average fields, eq. (2.8), which enter the above equation. 
As mentioned in the introduction, we could in principle vary all parameters of our phenomenological potentials $M(\boldsymbol{r}), V(\boldsymbol{r})$ and $S(\boldsymbol{r})$ in order to obtain the minimal binding energy. As this is not practically feasible, we restricted ourselves - except for some testing variations presented in sect. 4 - to one main optimization of the parameters which we present in the following along with some other corrections.

\subsection{THE VARIATION OF THE POTENTIAL PARAMETERS}

Altogether we have in the phenomenological approach about 21 free parameters, if we include here the charge density also as characterised by three numbers depth, radius and diffusivity. They should be varied independently, and ideally we would take the set which minimizes the total energy at a total density which we call in the following the saturation density. Apart from the fact that most of the parameters are determined from considerations and empirical facts which go beyond this paper, we can account for most of the necessary, however small changes in an approximate and global way. Assume that we change the scale of the radial dimension by a factor $s$ being close to one, i.e.

The wave functions transform then as

$$
\boldsymbol{r} \rightarrow \boldsymbol{r} / s
$$

$$
\phi_{i}(\boldsymbol{r}) \rightarrow \phi_{i}^{\prime}\left(\boldsymbol{r}^{\prime}\right)=s^{\frac{3}{2}} \phi_{i}(\boldsymbol{r}),
$$

since the new wave functions should be normalized as well. If we insert the new wave functions into the definition of the densities, eq. (2.2), and into the energy functional eq. (2.4), we obtain

$$
E(s)=s E_{\mathrm{C}}+s^{2} E_{\mathrm{k}}+s^{3}\left(t_{0} D_{0}+x_{0} D_{x}\right)+s^{5}\left(t_{1} D_{1}+t_{2} D_{2}+W_{0} D_{w}\right)+s^{6}\left(t_{3} D_{3}\right) .
$$

Here, we denote the Coulomb and the kinetic energy by $E_{\mathrm{C}}$ and $E_{\mathbf{k}}$, respectively, and introduce the invariant integrals $D_{0} \ldots D_{w}$ whose definitions are obvious by comparing eq. (2.19) with eq. (2.4), e.g.

$$
D_{0}=\frac{1}{2} \int \mathrm{d} r\left\{\rho^{2}(r)-\rho_{\mathrm{n}}^{2}(\boldsymbol{r})-\rho_{\mathrm{p}}^{2}(\boldsymbol{r})\right\} .
$$

The optimal value $s_{0}$ of the scaling factor is then simply given by

$$
\left.\frac{\partial E}{\partial s}\right|_{s=s_{0}}=0
$$

leading to an improved scale corrected value of the energy, i.e.

$$
E_{0}=E\left(s=s_{0}\right) \text {. }
$$

The scale correction can be applied also, if we work with deformed phenomenological potentials. It replaces there in a quantum-mechanically meaningfull way the otherwise only vaguely defined "volume conservation". The latter has a definite meaning only in the context of the liquid-drop model from which it actually has been borrowed. 


\subsection{THE OCCUPATION OF THE SINGLE-PARTICLE STATES}

In a self-consistent calculation the occupation is trivial as the lowest total energy is given by the $Z$ or $N$ single-particle wave functions with the lowest eigenvalues $\varepsilon_{i}$. For the phenomenological wave functions, this need not necessarily be true with respect to the single-particle energies $e_{i}$. In this case, the requirement for the optimal occupation must be the following,

$$
\Delta E_{i k}=\left\langle H\left(\rho-\rho_{i}+\rho_{k}\right)-H(\rho)\right\rangle>0
$$

for all occupied states $|i\rangle$ and all empty states $|k\rangle$. This statement is, of course, identical with the requirement that the energy is a minimum with respect to all possible occupations. As these "particle-hole excitations" $\Delta E_{i k}$ are approximately given by

$$
\Delta E_{i k} \approx-r_{i}+r_{k},
$$

it is sufficient to investigate only a few states very close to the Fermi energy. The optimization (2.23) can be done by an iterative procedure, which however costs some computer time, since the invariant integrals $D_{i}$ defined in eq. (2.19) have to be calculated anew for each occupation. In the practical applications we have seen that the inclusion of pairing effects, which we describe in the following and which is numerically much faster than the reoccupation procedure, has almost the same result of lowering the energy by a similar amount.

In both the self-consistent and the phenomenological approach, the total energy as defined by eq. (2.4) has to be corrected for a number of shortcomings connected to the relatively poor ansatz of a Slater determinant for the ground-state wave function. We describe here some of these corrections.

\subsection{SHORT RANGE RESIDUAL INTERACTIONS}

It has been shown by Vautherin ${ }^{10}$ ) that pairing correlations can be included in the HF procedure defined by eqs. (2.2), (2.7), (2.8) by doing a BCS calculation at the end of each iteration. We can do the same in our approach in terms of the single-particle energies $e_{i}$. Thereby the occupation numbers $n_{i}$ in eq. (2.2) have to be identified with the usual BCS occupation numbers,

$$
n_{i}=\frac{1}{2}\left[1-\frac{e_{i}-\lambda}{E_{i}}\right], \quad E_{i}=\sqrt{\left(e_{i}-\lambda\right)^{2}+\Delta^{2}} .
$$

The Fermi energy $\lambda$ and the pairing gap $\Delta$ are the solutions of the BCS equations

$$
\frac{2}{G}=\sum_{i}^{\prime} \frac{1}{E_{i}}, \quad N=\sum_{i} n_{i} .
$$

Following a suggestion of Strutinsky ${ }^{3}$ ), the coupling constant $G$ and the cut-off in the summation $\sum$ ' are related to an "average pairing gap" $\tilde{\Delta}$; for details and the values of parameters we refer to the literature ${ }^{4,6}$ ). Except for the introduction of the 
occupation numbers $n_{i}$, one has to add the usual pair condensation energy,

$$
E_{\mathrm{p}}=-\Delta^{2} / G
$$

to the total energy, eq. (2.1).

The inclusion of the pairing effects is mostly a smoothing of the total energy as a function of deformation or of nuclear numbers. It represents an alternative to the reoccupation procedure described above - although the physical assumption lying behind the two effects are of different nature - and leads to a similar, and not very significant modification of the total energy.

\subsection{CENTER-OF-MASS AND ROTATIONAL CORRECTIONS}

It is well-known that a product wave function of the Slater type is not an eigenfunction of the total momentum operator $\boldsymbol{P}$ nor of the operator of the total angular momentum $\boldsymbol{J}$. This shortcoming results in spurious contributions to the total energy, which however can be projected out. In a perturbative approach, one obtains a correction $\Delta E_{\text {c.m. }}$ for the spurious c.m. motion

$$
\Delta E_{\mathrm{c} . \mathrm{m} .}=-\frac{1}{2 M}\left\langle\boldsymbol{P}^{2}\right\rangle
$$

where $M$ is the total mass of the nucleus $M-m A$. Similarly, the spurious rotational energy is subtracted with the correction

$$
\Delta E_{\mathrm{rot}}=-\left\langle\boldsymbol{J}^{2}\right\rangle / 2 \mathscr{J} .
$$

which is only a good approximation, however, for an ideal rotational nucleus. In eq. (2.28), $\mathscr{J}$ is the moment of inertia of the nucleus. The expectation values $\left\langle\boldsymbol{P}^{2}\right\rangle$ and $\left\langle\boldsymbol{J}^{2}\right\rangle$ are evaluated as two-body matrix elements between the Slater determinants and the moment of inertia can be obtained with the cranking model ${ }^{4,21}$ ), both including pairing.

Our final expression of the total energy is thus given by

$$
E=\left.\int H(\boldsymbol{r}) \mathrm{d} \boldsymbol{r}\right|_{s=s_{0}}+\Delta E_{\text {c.m. } .}+\Delta E_{\mathrm{rot}}+E_{\mathrm{p}}
$$

We finally mention a possible source of numerical errors which is connected to the solution of both the HF equation (2.7) and the phenomenological Schrödinger equation (2.10). This solution is usually performed by expansion of the eigenfunctions in deformed harmonic oscillator basis and matrix diagonalization ${ }^{10,22}$ ). For practical reasons, this basis has to be truncated. It is reasonable to include those oscillator states $\left(n_{z}, n_{\rho}, A\right)$ which obey the cut-off relations

$$
\begin{gathered}
\left(n_{z}+\frac{1}{2}\right) q^{-\frac{z}{2}}+\left(2 n_{\rho}+|\Lambda|+1\right) q^{\frac{1}{3}} \leqq N_{E}+\frac{3}{2}, \\
n_{z}+2 n_{\rho}+|\Lambda| \leqq N_{0},
\end{gathered}
$$


where $q$ is the ratio of the two oscillator frequencies $\omega_{\rho}, \omega_{z}$ for axially symmetric shapes with a constant volume

$$
q=\frac{\omega_{\rho}}{\omega_{z}}, \quad \hbar \omega_{0}=\hbar\left(\omega_{\rho}^{2} \omega_{z}\right)^{\frac{1}{3}}=C A^{-\frac{1}{3}}(\mathrm{MeV}) .
$$

As a consequence of this truncation, the results of the total binding energy might depend on the parameters $h \omega_{0}$ and $q$, and have therefore to be minimized with respect to these parameters. This is done in the HF calculations by Flocard et al. ${ }^{11,12}$ ). The optimization of $\hbar \omega_{0}$ amounts practically to the same as the scaling correction (2.1) described above. The parameter $q$ is fixed by the nuclear shape in our approach as described by Damgaard et al. ${ }^{22}$ ). We shall not vary $q$ in the following calculations as their choice works well in the Strutinsky calculations. However, we keep in mind that some variations in $q$ may be necessary in our calculations.

\section{Results}

Without going into more details of our calculations, we want to present in this section some results of binding and deformation energies of various nuclei using various effective interactions. A detailed discussion of some of the corrections mentioned in the last section will be given along with illustrative figures in sect. 4. In some of the cases presented here, we compare our results to those obtained either in a self-consistent CHF calculation or a Strutinsky calculation.

We use five different sets of parameters of the Skyrme interaction; they are listed in table 1. SKY I and II are the "classical" versions introduced by Vautherin and Brink ${ }^{8}$ ). SKY III is a newer version mainly used in the most recent publication by Flocard et al. $\left.{ }^{11}\right)$. For historical reasons, we have in some of the calculations also used the set SKY X, which differs from SKY III only in the omission of the exchange part of the Coulomb interaction; it gives almost identical results to those of SKY III. All these parameter sets have been determined, by the Orsay group, by fitting the HF results to experimental binding energies and radii of spherical nuclei. This is not the case for the set SKY T, which we have tried here temporarily to fit the fission barrier of ${ }^{240} \mathrm{Pu}$ in our approximation.

In one case (see fig. 3) we have also used the interaction of Negele in the density matrix expansion (DME) which can be handled in a very similar way as the Skyrme interaction ${ }^{9}$ ).

Since the pure parameters of the Skyrme interaction might not be very instructive for the untrained eye, we list in table 2 the nuclear matter properties corresponding to these parameter sets.

The phenomenological average potential which we have used in all cases is the newest version ${ }^{6}$ ) of the deformed Woods-Saxon potential originally introduced by Damgaard $e t a l^{22}$ ) and used in many Strutinsky calculations ${ }^{4}$ ). The parameters are listed in table 3. 
TABLE 1

Different sets of parameters of the Skyrme interaction

\begin{tabular}{lcccccc}
\hline & $\begin{array}{c}t_{0} \\
\left(\mathrm{fm}^{3} \cdot \mathrm{MeV}\right)\end{array}$ & $\begin{array}{c}x_{0} \\
\left(\mathrm{fm}^{3} \cdot \mathrm{MeV}\right)\end{array}$ & $\begin{array}{c}t_{1} \\
\left(\mathrm{fm}^{5} \cdot \mathrm{MeV}\right)\end{array}$ & $\begin{array}{c}t_{2} \\
\left(\mathrm{fm}^{5} \cdot \mathrm{MeV}\right)\end{array}$ & $\begin{array}{c}t_{3} \\
\left(\mathrm{fm}^{6} \cdot \mathrm{MeV}\right)\end{array}$ & $\begin{array}{c}W_{0} \\
\left(\mathrm{fm}^{5} \cdot \mathrm{MeV}\right)\end{array}$ \\
\hline SKY I & -1057.3 & 0.56 & 235.9 & -100.0 & 14463.5 & 120.0 \\
SKY II & -1169.9 & 0.34 & 585.6 & -27.0 & 9331.1 & 105.0 \\
SKY III & -1128.75 & 0.45 & 395.0 & -95.0 & 14000.0 & 120.0 \\
SKY X & -1133.40 & 0.49 & 395.0 & -95.0 & 14000.0 & 120.0 \\
SKY T & -1144.7 & 0.07 & 402.1 & 56.1 & 9656.3 & 131.1 \\
\hline
\end{tabular}

SKY I and SKY II are from ref. ${ }^{8}$ ). SKY III is from refs. ${ }^{11,{ }^{2}}$ ). SKY X is the same as SKY III if the exchange part of the Coulomb interaction is omitted. SKY $T$ is tried in this paper to fit to a lower fission barrier of ${ }^{240} \mathrm{Pu}$.

TABLE 2

Nuclear matter properties given by the Skyrme interactions

\begin{tabular}{lccccc}
\hline & $\begin{array}{c}k_{\mathrm{F}} \\
\left(\mathrm{fm}^{-1}\right)\end{array}$ & $\begin{array}{c}E / A \\
(\mathrm{MeV})\end{array}$ & $\begin{array}{c}K \\
(\mathrm{MeV})\end{array}$ & $\begin{array}{c}a_{\tau} \\
(\mathrm{MeV})\end{array}$ & $m^{*}$ \\
\hline SKY I & 1.32 & -15.98 & 370.5 & 29.2 & 0.91 \\
SKY II & 1.30 & -16.00 & 342.0 & 34.1 & 0.58 \\
SKY III & 1.29 & -15.84 & 353.6 & 28.2 & 0.76 \\
SKY X & 1.29 & -16.09 & 352.1 & 30.0 & 0.76 \\
SKY T & 1.30 & -15.39 & 338.3 & 25.0 & 0.60 \\
\hline
\end{tabular}

$k_{\mathrm{F}}$ is the Fermi momentum; $E / A$ is the binding energy per particle; $K$ is the compression modulus; $a_{\mathfrak{t}}$ is the symmetry energy; $m^{*}$ is the effective mass.

TABLE 3

Parameters of the phenomenological average fields

\begin{tabular}{ll}
\hline Input: & $\bar{\delta}=\left(I+0.0112 Z^{2} A-\frac{5}{3}\right) /\left(1+3.15 A^{-\frac{1}{3}}\right), \quad I=(N-Z) / A$ \\
$\bar{\varepsilon}$ & $=-0.147 A^{-\frac{1}{3}+0.330 \bar{\delta}^{2}+0.00248 Z^{2} A^{-\frac{4}{3}}}$ \\
$V_{\mathrm{s}}$ & $=-53.6 \mathrm{MeV}, \quad V_{\mathrm{d}}=-45.0 \mathrm{MeV}$ \\
$r_{0}$ & $=1.16 \mathrm{fm}, \quad a_{\mathrm{v}}=0.66 \mathrm{fm}, \quad a_{\mathrm{s}}=0.55 \mathrm{fm}$ \\
\hline
\end{tabular}

Mass field $M(r)$

$$
M_{0}=0
$$

Charge distribution Spin-orbit field $S(\boldsymbol{r})$

$$
\begin{aligned}
& Z_{0}=12 \\
& R_{0}=r_{0} A \frac{1}{3}(1+\bar{\varepsilon}) \\
& R_{\mathrm{s}}=R_{0}\left[1-\frac{1}{3} \pi^{2}\left(\frac{a_{\mathrm{s}}}{R_{\mathrm{O}}}\right)^{2}\right]
\end{aligned}
$$

$R_{\mathrm{ch}}=r_{0} A^{\frac{1}{3}}\left[1-\frac{1}{3}(I-\bar{\delta})\right]$
Central field $V(\boldsymbol{r})$

$$
\begin{aligned}
V_{0, Z(N)} & =V_{\mathrm{s}} \pm \bar{\delta} V_{\mathrm{d}} \\
R_{\mathrm{v}, Z(W)}{ }^{(0)} & =R_{0}-0.56 / R_{0}+0.82 \pm 0.22 \bar{\delta} \\
R_{\mathrm{v}} & =R_{\mathrm{v}}{ }^{(0)}\left[1-\frac{1}{3} \pi^{2}\left(\frac{a_{\mathrm{v}}}{R_{\mathrm{v}}{ }^{(0)}}\right)^{2}\right]
\end{aligned}
$$

Up to $V_{\mathrm{s}}$ and $V_{\mathrm{d}}$, the parameters are the same as given by Myers ${ }^{22}$ ); they are identical to ref. ${ }^{6}$ ). If the symbols carry no reference to either $Z$ or $N$, they hold for both protons and neutrons.

Errata: In ref. $\left.{ }^{6}\right)$, eq. (45) should read as $R_{0}=r_{0} A \frac{1}{3}(1 \div \bar{\varepsilon})$ and eq. $(47)$ as $V_{0, z(N)}=-53.6(-)$ $45.0 \bar{\delta} \mathrm{MeV}$. 
The Schrödinger equation (2.10) which defines our single-particle wave functions is solved by expansion in a deformed harmonic oscillator basis as described in detail by Pauli ${ }^{6}$ ). Unless otherwise mentioned, we use cut-off parameters $N_{E}=10, N_{0}=20$ in spherical nuclei and $N_{E}=14, N_{0}=20$ in deformed nuclei, the definitions of the cut-off were given in eq. (2.30). We have only considered axially symmetric shapes $(\alpha=0)$ in the $(c, h, \alpha)$ parameterization of Damgaard et al. $\left.{ }^{22}\right)$. Accordingly, the densities (2.2) are evaluated in cylindrical coordinates $(z, \rho)$. For the detailed formulae we refer to the appendix. The numerical integrations of the energy density eq. (2.4) are performed using a 24-point Gauss-Hermite formula in the $z$-direction and a 10-point Gauss-Laguerre formula in the $\rho$-direction.

\subsection{SPHERICAL NUCLEI}

We have calculated the binding energies and $\mathrm{rms}$ radii of the spherical nuclei ${ }^{16} \mathrm{O}$, ${ }^{40} \mathrm{Ca},{ }^{48} \mathrm{Ca},{ }^{90} \mathrm{Zr}$ and ${ }^{208} \mathrm{~Pb}$. The rms radii are defined as

$$
\begin{aligned}
& r_{\mathrm{p}}=\sqrt{\left(\left\langle z^{2}\right\rangle_{\mathrm{p}}+\left\langle\rho^{2}\right\rangle_{\mathrm{p}}\right) / Z}, \\
& r_{\mathrm{n}}=\sqrt{\left(\left\langle z^{2}\right\rangle_{\mathrm{n}}+\left\langle\rho^{2}\right\rangle_{\mathrm{n}}\right) / N}, \\
& r_{\mathrm{m}}=\sqrt{\left(\left\langle z^{2}\right\rangle+\left\langle\rho^{2}\right\rangle\right) / A},
\end{aligned}
$$

for the proton, neutron and total nucleon distributions, respectively.

We have also calculated the proton charge radii of these nuclei from $r_{p}$ by correcting the c.m. motion and the finite size of proton according to the formula ${ }^{9,16}$ )

$$
r_{\mathrm{c}}^{2}=r_{\mathrm{r}}^{2}+\left\langle r^{2}\right\rangle_{\text {protan }}-\frac{3}{2} \frac{b_{\mathrm{p}}^{2}}{A}
$$

where $b_{\mathrm{p}}$ is the oscillator length defined by

$$
b_{\mathrm{p}}=\left(\hbar / m \omega_{0}\right)^{\frac{1}{2}} .
$$

For the rms radius of the proton $\left\langle r^{2}\right\rangle_{\text {proton }}$, we use the experimental value $0.8 \mathrm{fm}$.

The results are shown in table 4; they are all scale corrected according to eqs. (2.17)-(2.22). SM denotes our results obtained with the Woods-Saxon wave functions; they are compared with the available self-consistent results (HF) from $\mathrm{HF}$ calculations. The overall agreement with both the HF results and the experimental numbers is quite satisfactory. The agreement of the Woods-Saxon radii with experiment is of the same quality as that of the self-consistent ones. This is not surprising, since the Woods-Saxon parameters are fitted to experimental radii. The binding energies are generally slightly less than the HF results; this is obviously due to the missing selfconsistency. However, the discrepancies are only about $100 \mathrm{keV}$ per particle in the heavier nuclei and less than $\approx 300 \mathrm{keV}$ per particle in the lighter nuclei. The parameter set SKY T, gives the nicest agreement of our results with the experimental numbers. However, HF calculations with this interaction lead to too low energies. 


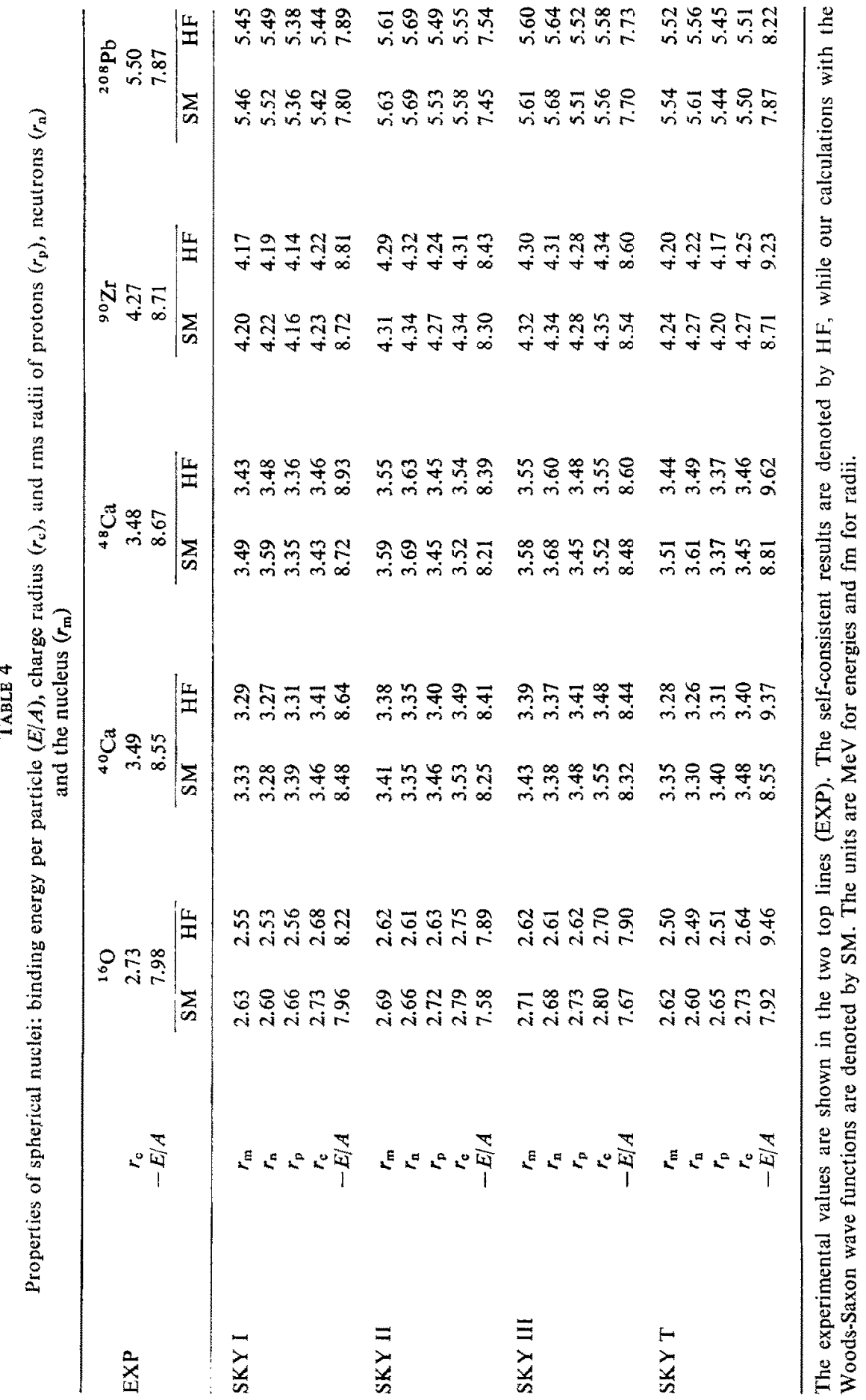


Next we have evaluated the single-particle removal energies $r_{i}$ [eq. (2.16)] of ${ }^{208} \mathrm{~Pb}$. In figs. 1 and 2 they $(\mathrm{SM})$ are shown together with the self-consistent separation energies (HF). To the left of the figures, the experimental s.p. energies (EXP) and the eigenvalues $e_{i}$ [eq. (2.10)] of the Woods-Saxon potential (WS) are shown. It is evident from these figures that our approximation is able to reproduce the typical features of the self-consistent spectra. For all interactions the difference between $r_{i}$

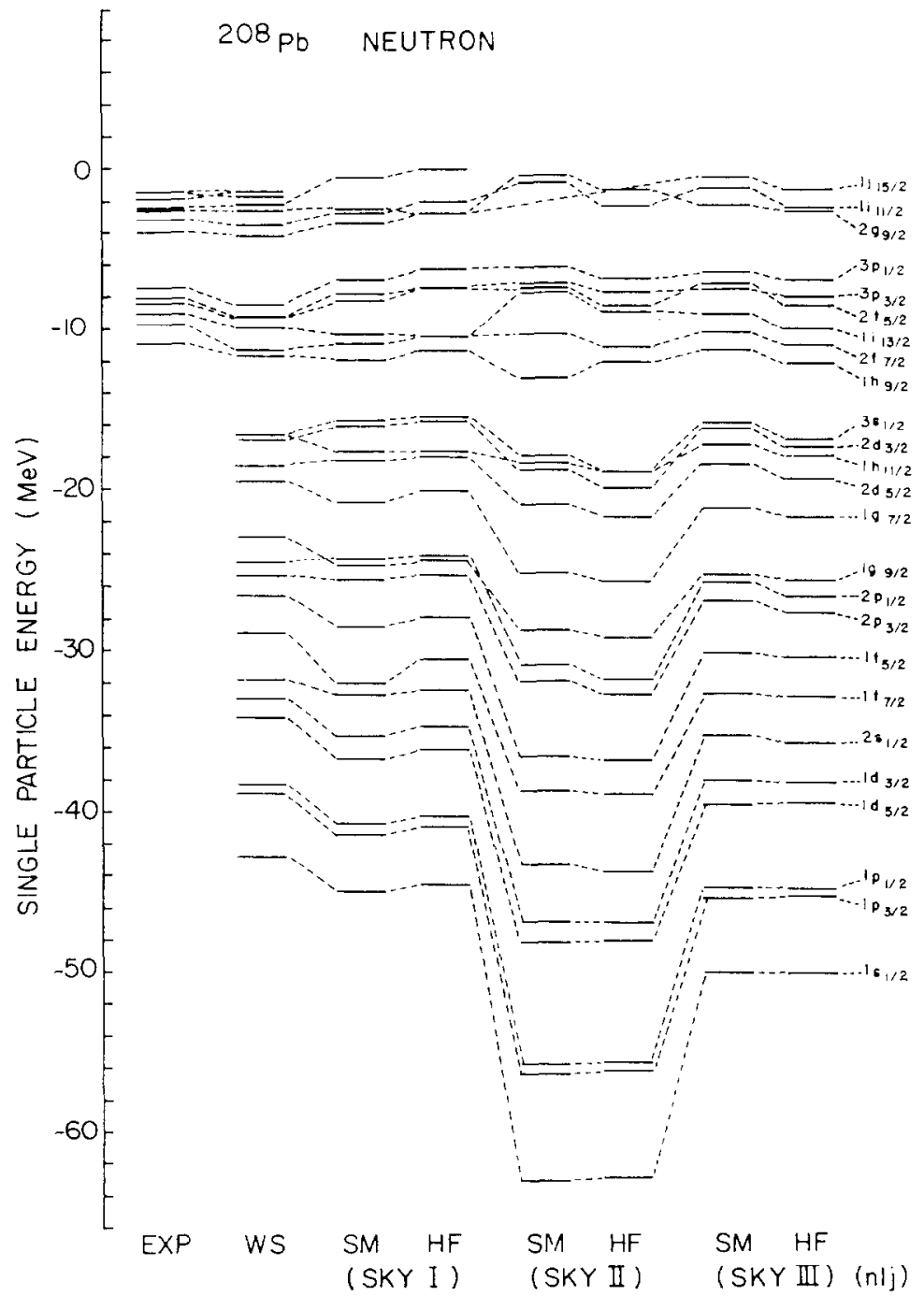

Fig. 1. Neutron single-particle energies of ${ }^{208} \mathrm{~Pb}$ (in MeV). EXP and WS refer to the experimental single-particle energies and the eigenvalues of the Woods-Saxon potential, respectively. With the Skyrme interactions, the Hartree-Fock self-consistent single-particle energies are denoted by $\mathbf{H r}$, while the removal energies obtained with the Woods-Saxon single-particle wave functions are denoted by SM. 


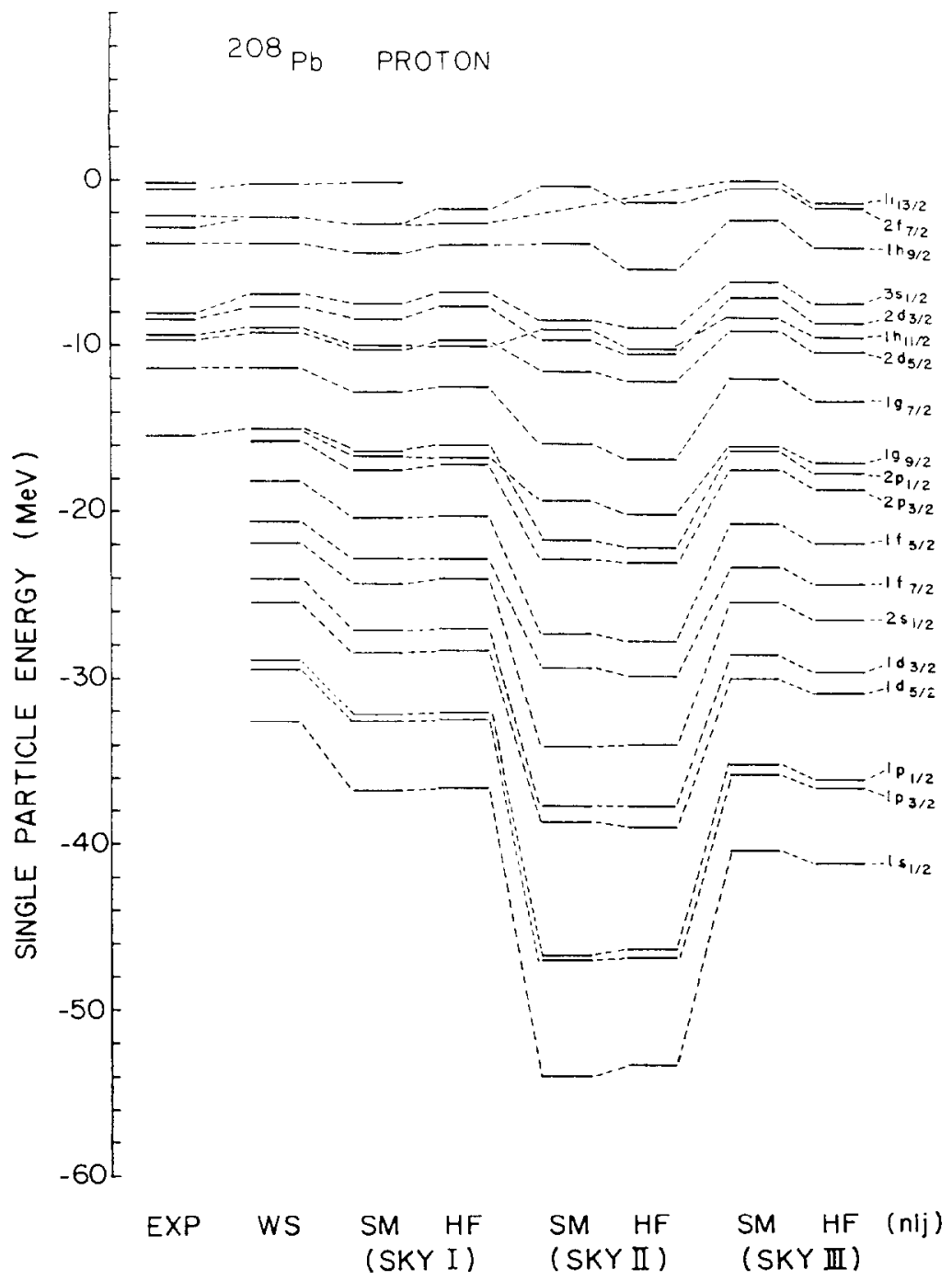

Fig. 2. Proton single-particle energies of ${ }^{208} \mathrm{~Pb}$. Notations as in fig. 1.

and $\varepsilon_{i}$ is less than $\approx 1 \mathrm{MeV}$, whereas both of them differ from the Woods-Saxon levels by much more, especially the low-lying levels in the case of SKY II and SKY III. The removal energies $r_{i}$ reflect also the known deficiency of the Skyrme interactions that they do not bind all experimentally seen hole states ${ }^{8}$ ). The agreement with experiment is slightly better for the $r_{i}$ than for the self-consistent $\varepsilon_{i}$; this again is due to the fact that the Woods-Saxon potential has been fitted to the single-particle spectrum of ${ }^{208} \mathrm{~Pb}$.

Summing up, we can say that our approximation to binding and removal energics and scaled radii comes rather close to the self-consistent results. It will therefore be interesting to see the behaviour of the energy as a function of deformation. 


\subsection{ONE-DIMENSIONAL DEFORMATION ENERGY CURVES}

To obtain the first guess of deformation energy curves, we calculate the total binding energy for deformed shapes of the Woods-Saxon potential along the line $h=\alpha$ $=0$. This line has been fitted approximately to the liquid-drop valley in the $(c, h)$ space for medium and heavy nuclei [see refs. ${ }^{6,22}$ )] and is thus a rough approximation to the fission path. Although this definition might not be justified in a light nucleus as ${ }^{40} \mathrm{Ca}$, we compute the deformation energies of ${ }^{40} \mathrm{Ca}$ for some interactions along this family of shapes. Fig. 3 shows the total energy as a function of the elongation parameter $c$. Two of the curves are obtained with Skyrme interactions, the top curve is calculated with the Negele-DME force. Apart from a constant shift, the curves behave in a very similar way. The energies are not scaled here; in the Negele interaction the scaling cannot be applied due to the more complicated density dependence of this interaction. Since no residual interaction has been included, we can see the level crossings appearing as cusps in the curves.

In order to compare with a self-consistent result, we perform a constrained HartreeFock (CHF) calculation with the force SKY X; thereby the original code of Vautherin $\left[\right.$ ref. $\left.\left.{ }^{10}\right)\right]$ is used after a modification which allows fixed configuration of the levels to be occupied. The total quadrupole moment $Q_{2}$ is fixed with a linear constraint. The result is shown in fig. 4 . The top curve is the same as the corresponding one in fig. 3 ,

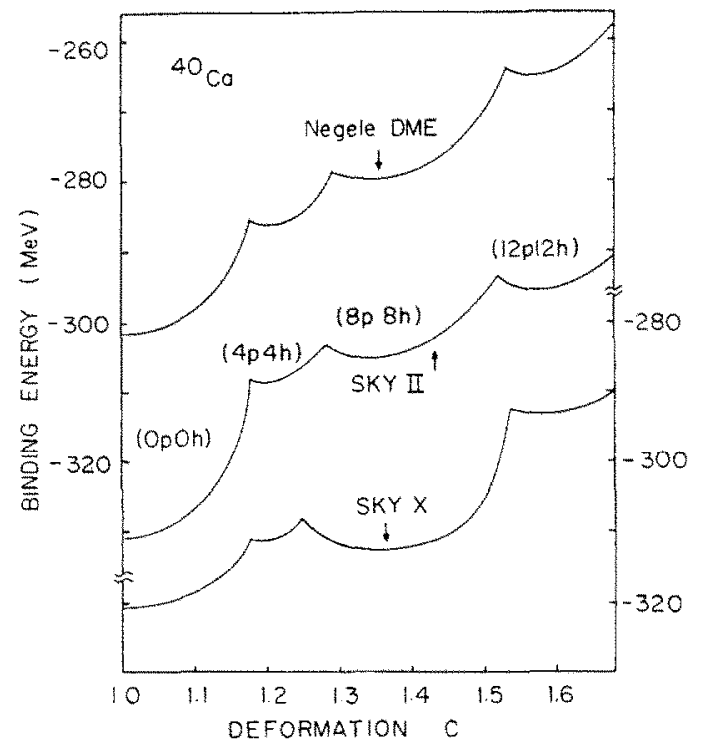

Fig. 3. Deformation energy curves of ${ }^{40} \mathrm{Ca}$ obtained with the expectation-value method for axially symmetric shapes $h=\alpha=0$. Besides two versions of the Skyrme interaction (SKY II, X) we also show a curve obtained with Negele's interaction in the density matrix expansion (DME). The cusps occur when the single-particle energies cross. $(n \mathrm{p} n \mathrm{~h})$ denotes the particle-hole excitations after these crossings, taking the spherical ${ }^{40} \mathrm{Ca}$ core as a reference $(0 \mathrm{p} 0 \mathrm{~h})$. The corresponding crossings of proton and neutron levels occur nearly at the same deformations. The basis is not scaled and the cut-off parameters are $N_{\mathrm{o}}=18$ and $N_{E}=8$. 


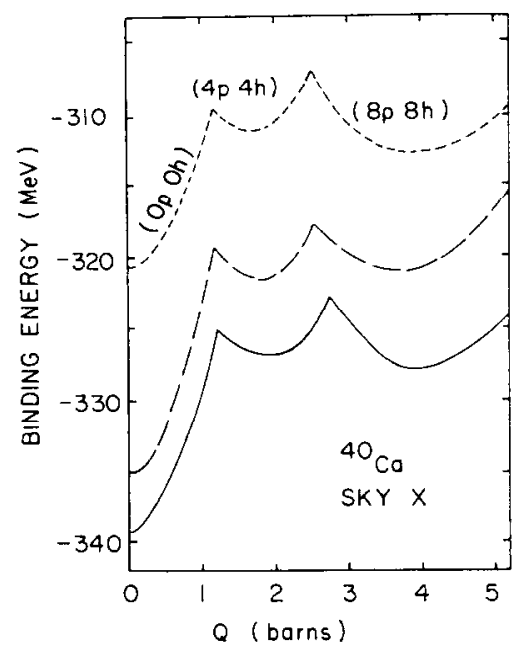

Fig. 4. Deformation energy curves of ${ }^{40} \mathrm{Ca}$ as a function of the quadrupole moment, using the interaction SKY X. The top curve corresponds to the lowest curve in fig. 3. The curve in the middle is obtained after scaling of the basis. The lowest curve is the self-consistent result obtained by a HF calculation with a fixed configuration $(n \mathrm{p} n \mathrm{~h})$. At non-equilibrium points of the self-consistent curve, a linear constraint of the quadrupole moment is used. The basis parameters $\hbar \omega_{0}$ and $q$ are optimized in all branches of the self-consistent curve.

but here it is plotted as a function of the quadrupole moment $Q_{2}$ which was calculated from the wave functions at each deformation $c$. The curve in the middle is the same after the scaling. We see that this procedure brings the total curve down by $8-14 \mathrm{MeV}$ and is therefore very important for the absolute values of the energy. The lowest curve is the self-consistent deformation energy curve. In the ground state, we are only missing $\approx 4 \mathrm{MeV}$ of the self-consistent binding energy. With increasing deformation, the discrepancy increases gradually to reach $\approx 8 \mathrm{MeV}$ at $Q_{2}=5 \mathrm{~b}$.

One reason for this increasing difference might be the fact that the energies obtained with the Woods-Saxon wave functions have not been minimized with respect to other degrces of frccdom $\left(Q_{4}, Q_{6}\right.$ etc. $)$, a feature which is automatically guaranteed in the $\mathrm{CHF}$ calculations. Another reason could be connected to the choice of the parameters of the harmonic oscillator basis (see eq. (2.31)). In the CHF calculation, both parameters $\hbar \omega_{0}$ and $q$ have been optimized for each value of $Q_{2}$. In the expectation-value method, the scaling is closely related to the optimization of $\hbar \omega_{0}$. The parameter $q$ has not been varied in our calculations, its values are determined in the way described by Damgaard et al. ${ }^{22}$ ).

Apart from this increase of the defect in the total energy, the agreement of our results with the HF result is rather remarkable. Note especially that the positions of the local minima of the curves closely coincide. This result had already been obtained for heavier nuclei in our preliminary work ${ }^{1}$ ). Thus our method allows at least in a semiquantitative manner to calculate deformation energy curves and to study the effect of different interactions. 
To test the method in a heavy nucleus, we choose the standard case ${ }^{240} \mathrm{Pu}$. Fig. 5 shows the one-dimensional deformation energies of ${ }^{240} \mathrm{Pu}$, again taken along the LD valley $h=0$, for four Skyrme interactions. Here we have included pairing interactions with a constant average gap $\tilde{\Delta}$ as described in sect. 2 above. All curves show a ground-state minimum around $c=1.12$, and a second minimum around $c=1.4$. On the average, except SKY T, the deformation energy increases however much too rapidly, leading to a second fission barrier of the order of $\approx 30 \mathrm{MeV}$. The further increase at $c \geqq 1.7$ is due to the fact that the energy is not minimized with respect to $h \neq 0$; (see subsect. 3.3 below). Since we expect from Strutinsky calculations ${ }^{4}$ ) that the second barrier should be close to $h=0$, this deficiency cannot be wholly due to the missing optimization in the degree of freedom perpendicular to the fission degree. Apart from the possible inadequacy of the basis deformation $q$ chosen, which we mentioned above, one might conjecture from this result that the liquid-drop properties of the Skyrme interactions correspond to a too large surface-energy coefficient. To illustrate this, we show in fig. 6 three deformation energy curves obtained with the Strutinsky method using the same Woods-Saxon potential (along $h=0$ ) but three different values of the surface energy in the liquid-drop energy. We see from this that a value of $a_{\mathrm{s}} \approx 24$ would lead to similar curves as those displayed in fig. 5 . A conclusion for a surface energy coefficient of $a_{\mathrm{s}} \approx 24$ of the Skyrme interactions is however not justificd. First, a preliminary determination of this coefficient by Vautherin [ref. $\left.{ }^{10}\right)$ ] led to appreciably lower values $\approx 20.7$ and $\approx 22.0$ for the interactions SKY I and II, respectively. Second, a detailed investigation of the liquid-drop parts

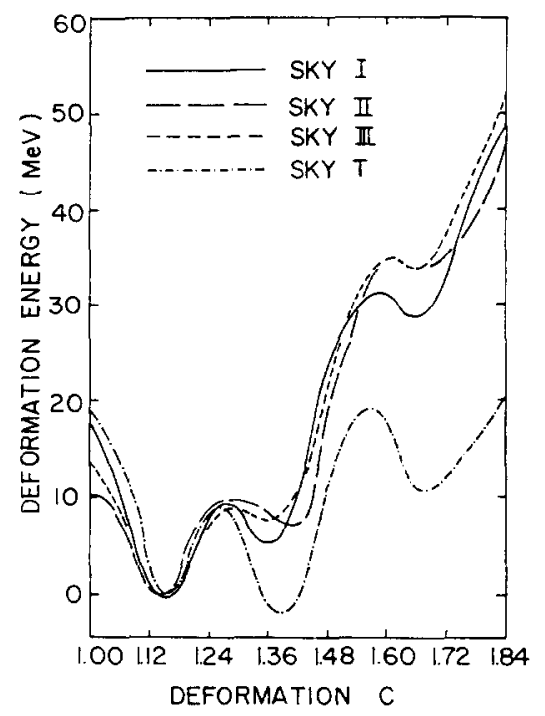

Hig. 5. Deformation energy curves of ${ }^{2 * 0} \mathrm{Pu}$ for axially symmetrical shapes along $h=\alpha=0$, ubtained with the expectation-value method. Pairing with constant $\tilde{A}$ is used; the basis is scaled; and the cut-offs are $N_{0}=20, N_{E}=14$. 


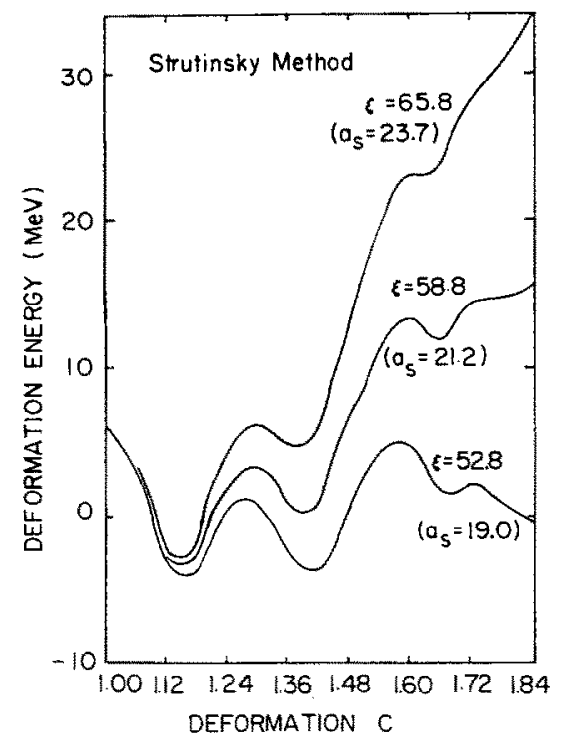

Fig. 6. Deformation energy curves of ${ }^{240} \mathrm{Pu}$ along $h=\alpha=0$, using the Strutinsky method. The liquid-drop deformation energy is calculated according to ref. ${ }^{29}$ ),

$$
E_{\mathrm{LD}}=C_{3} \frac{Z^{2}}{A^{\frac{2}{3}}}\left[\left(B_{\mathrm{C}}-1\right)+\frac{1}{2} \zeta \frac{A}{Z^{2}}\left(B_{\mathrm{s}}-1\right)\right]
$$

where $C_{3}=0.72$ and $\zeta=\xi\left(1-2.84 I^{2}\right), I=(N-Z) / A ; B_{\mathrm{s}}$ and $B_{\mathrm{C}}$ are the ratios of the surface and Coulomb energies to those at the spherical shape. Three different values of $\xi$ are used, the corresponding surface coefficient $a_{s}$ is given in the parentheses. Constant pairing is included in the shell-correction energies.

of self-consistent deformation energy curves obtained with SKY III ${ }^{14}$ ) suggests a value of $a_{\mathrm{s}}$ for this interaction which lies rather close to the value $a_{\mathrm{s}}=18.7$ given by Myers and Swiatecki ${ }^{24}$ ).

To see whether the truncation of the basis used might cause the drastic increase of deformation energies in fig. 5, we calculated a few points corresponding approximately to the ground-state minima $(c=1.18)$ and the outer saddle point $(c=1.60)$ with an increased number of shells included $\left(N_{E}=16\right.$ instead of 14). The resulting changes in the approximate barrier heights, shown in table 5, are however negligible and show that the truncation effects are too small to cause any serious errors.

\section{TABLE 5}

Approximate barrier heights along $h=\alpha=0, E_{\mathrm{B}}=E(c=1.6)-E(c=1.18)$, for ${ }^{240} \mathrm{Pu}$, evaluated for two different cut-off parameters

\begin{tabular}{lccc}
\hline$\left(N_{0}, N_{E}\right)$ & SKY I & SKY II & SKY III \\
\hline$(20,14)$ & 31.6 & 32.0 & 33.9 \\
$(22,16)$ & 30.2 & 31.1 & 32.8 \\
\hline
\end{tabular}


Before we compare our results for ${ }^{240} \mathrm{Pu}$ in more detail with the HF results, it is necessary to obtain a two-dimensional deformation energy surface, since we know from the Strutinsky results that the static fission path does not coincide exactly with the line $h=0$.

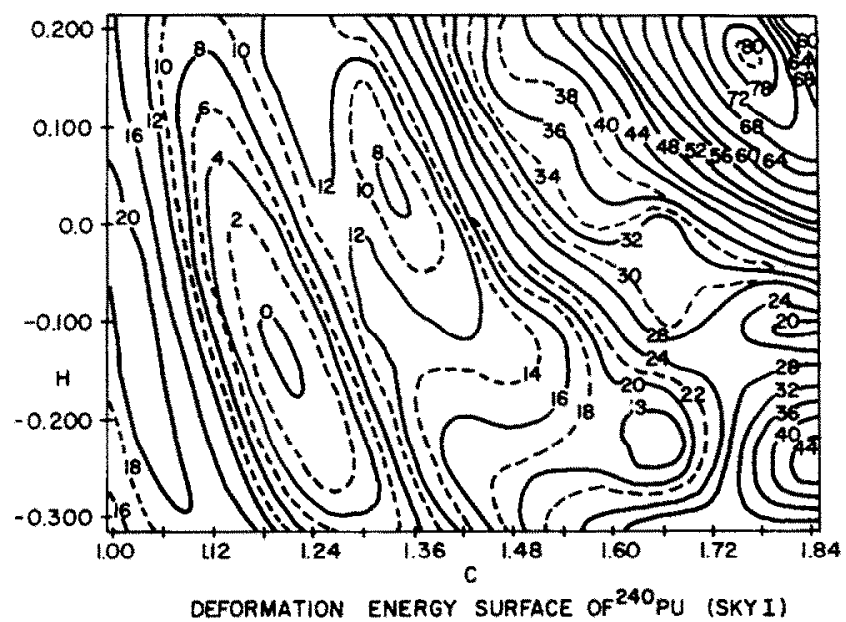

Fig. 7. Deformation energy surface of ${ }^{240} \mathrm{Pu}(\alpha=0)$ calculated by the expectation-value method using SKY I. The wave functions are obtained with cut-offs $N_{0}=20$ and $N_{E}=14$. Constant pairing and scaling are included. Numbers on the contour lines are in units of $\mathrm{MeV}$.

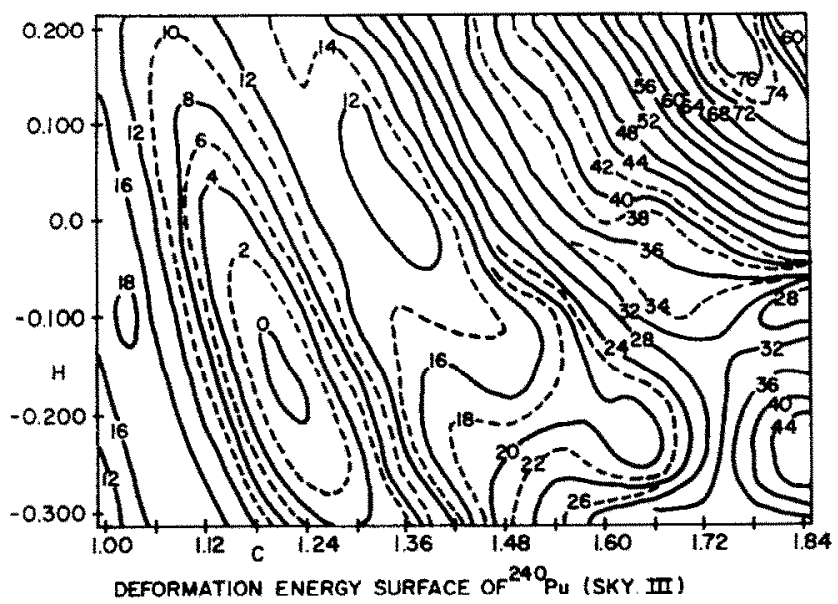

Fig. 8. Same as fig. 7 for the interaction SKY III.

\subsection{TWO-DIMENSIONAL DEFORMATION ENERGY SURFACES OF ${ }^{240} \mathrm{Pu}$}

As mentioned in the introduction, our method allows us to calculate multidimensional deformation energy surfaces with a still reasonable amount of work and computer time. We present in this section a few surfaces obtained for axially symmetric shapes $(\alpha=0)$ of our Woods-Saxon potential in the $(c, h, \alpha)$ parametrization. 
Figs. 7, 8 and 10 show the deformation energy surfaces of ${ }^{240} \mathrm{Pu}$ for the interactions SKY I, III and T respectively ${ }^{\dagger}$. We see that the degree of freedom $h$ brings the second saddle point down by $\approx 6-8 \mathrm{MeV}$ as compared to the case where $h=0$ (see fig. 5). The ground-state and isomer minima are also found at shapes with $h \neq 0$. The shape of the ground state with $c \approx 1.21$ and $h \approx-0.13$ is in excellent agreement with the results from Strutinsky calculations ${ }^{4,6}$ ).

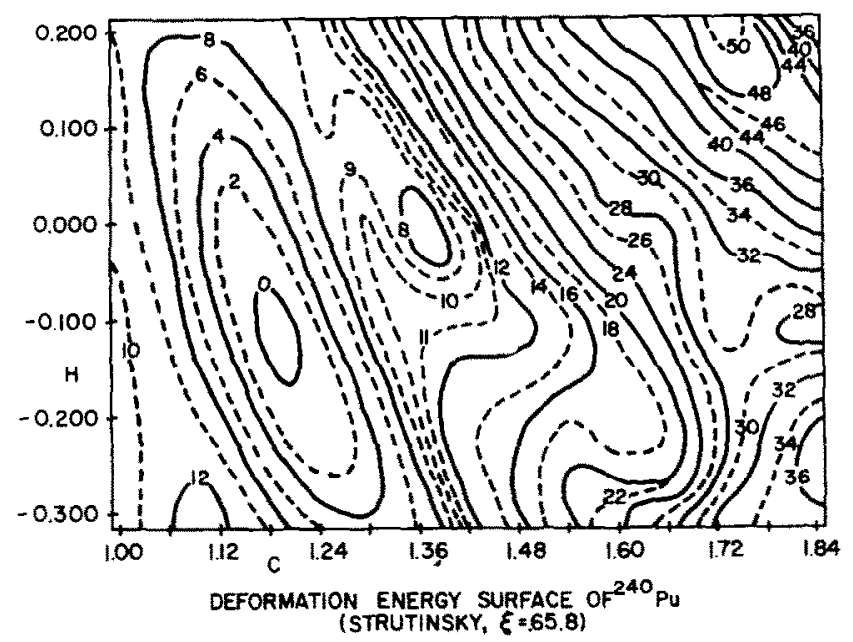

Fig. 9. Deformation energy surface of ${ }^{240} \mathrm{Pu}(\alpha=0)$ obtained with the shell correction method. The parameter $\xi$ of the liquid-drop energy, defined in the caption to fig. 6 , is 65.8 . Constant pairing is included. Numbers on the contour lines are in units of $\mathrm{MeV}$.

Apart from the fact that the fission barriers are much too high, as we already have seen in fig. 5 , the topology of these surfaces resembles much the one we know from the Strutinsky results. In order to illustrate this, we show in figs. 9 and 11 two Strutinsky surfaces for the same nucleus obtained with two different surface energy parameters but otherwise the same shell correction; the same Woods-Saxon potential is used as in all the other calculations.

Our result with SKY III is very close to the Strutinsky surface with the liquid-drop parameter $\zeta=65.8$ corresponding to a surface energy coefficient $a_{\mathrm{s}}=23.7$. Although this comparison should not be used to draw a conclusion about the liquid-drop behaviour of the Skyrme forces, as we have mentioned above in subsect. 3.2 , we can learn from it that the shell effects might be reproduced fairly well in our calculations and that it is the bulk part of the total energy which behaves wrong. From the newest HF results by Flocard $e t$ al. $^{12}$ ) we know that the fission barrier obtained with SKY III is less than half the value of the one found in fig. 8 (see also fig. 12 below). The wrong behaviour of the average part of the deformation energy seems thus to be mainly due to the lack of self-consistency.

* We omitted the surface obtained with SKY II, since it looks very similar to those with SKY I and SKY III. 
The fact that the shell-effects turn out to be more or less the same in all cases and also to the ones obtained with the shell-correction method leads us to the conclusion that they are much more connected to the shape of the average potential than to the details of the two-body interaction.

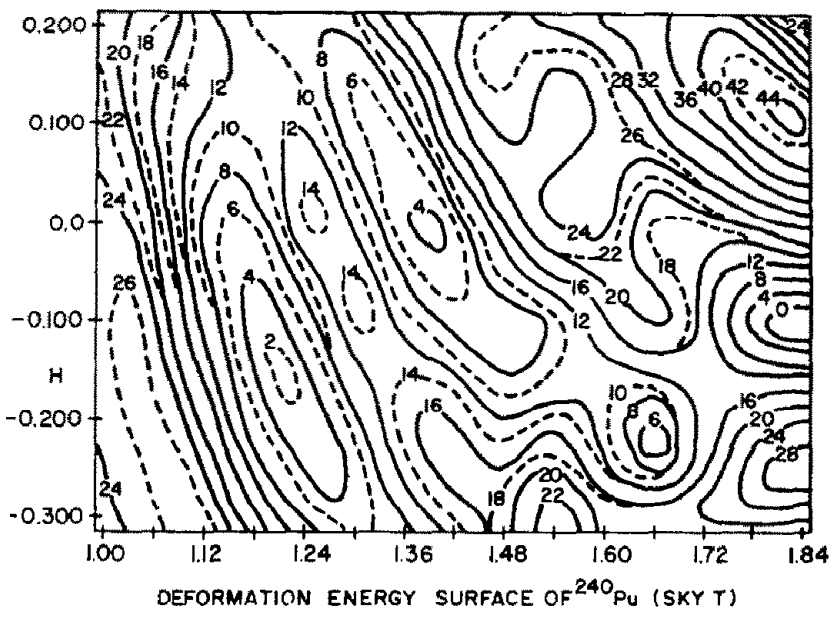

Fig. 10. Same as in fig. 7 for the interaction SKY T.

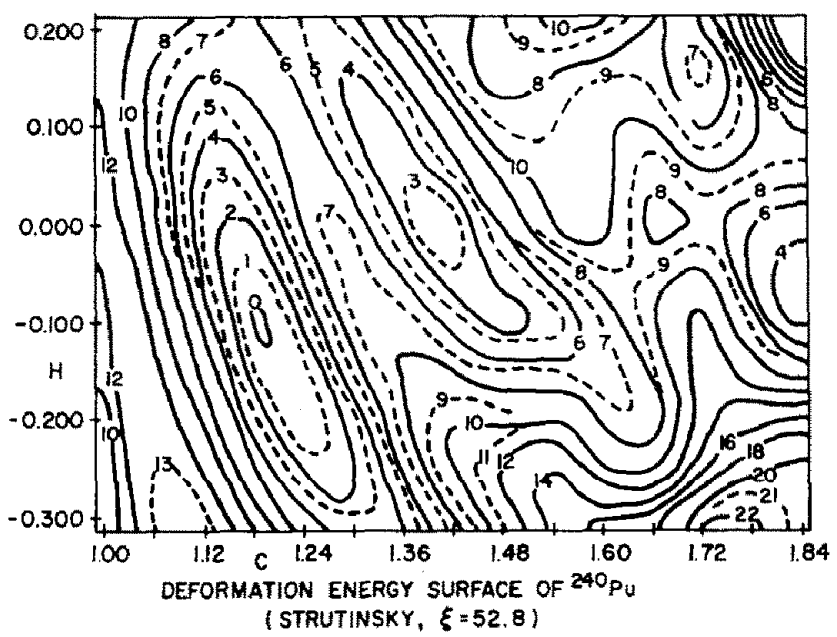

Fig. 11. Same as in fig. 9 with $\xi=52,8$.

So far, all available sets of Skyrme forces have been fitted to the properties of spherical nuclei. The variation of the values of these parameter sets indicates that there might be enough freedom to fit them to some deformation energies, too. We have tried here to do this with our expectation-value method. One preliminary version of parameters obtained in this way is the set " $T$ " in table 1 [ref. $\left.{ }^{25}\right)$ ]. With these 
parameters, we obtain indeed excellent results for the binding energies and radii of spherical nuclei, as presented above in table 4 , and at the same time considerably lower fission barriers of ${ }^{240} \mathrm{Pu}$, as shown in fig. 10 . The energy surface shown in this figure is indeed very close to the Strutinsky surface of fig. 11 which was obtained with the usual set of liquid-drop parameters.

The set SKY T of Skyrme parameters should, however, not be taken too seriously especially it is not thought to be used in self-consistent calculations as already shown in the case of spherical nuclei. In fact, some preliminary tests with the HF codes have indicated, that this interaction would give both too low ground-state energies

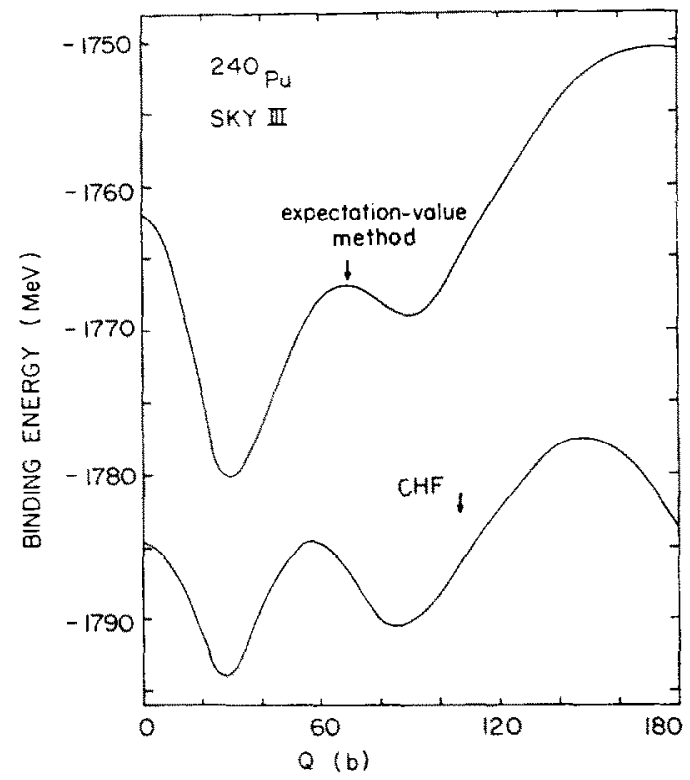

Fig. 12. Deformation energy of ${ }^{240} \mathrm{Pu}$ as a function of the quadrupole moment using the interaction SKY III. The upper curve is our result obtained from fig. 8 by minimizing the energy along lines of constant quadrupole moment. The lower curve is the HF self-consistent result using a quadratic constraint on the quadrupole moment.

and a too low fission barrier in a self-consistent treatment, as could be expected from the above results with the other interactions. The fit of this parameter set " $T$ " is rather thought to demonstrate that the Skyrme force with its six parameters is flexible enough to be adjusted to both spherical and deformed properties of nuclei.

For a detailed comparison of our results for ${ }^{240} \mathrm{Pu}$ with the self-consistent results, we should remember that in a CHF calculation the energy is automatically minimized with respect to all other deformation parameters except the one fixed by a constraint. For the force SKY III, the Orsay group has presented an energy-versus-quadrupole moment curve for the same pairing treatment as we use in the expectation-value calculations; i.e. with a constant value of the average gap $\tilde{\Delta}$ (and therefore constant 
pairing strengths $G_{\mathrm{n}}$ and $G_{\mathrm{p}}$ ). From our expectation-value results with SKY III in fig. 8, we first evaluate the lines of constant values of the quadrupole moment $Q_{2}$ and then for each value of $Q_{2}$, the energy is minimized. The curve so obtained is shown in fig. 12 (upper curve) and compared to the self-consistent result (lower curve).

We see from fig. 12 that the quadrupole moments found at the two minima coincide within $5 \%$ for the two curves. This confirmes the results found above for ${ }^{40} \mathrm{Ca}$ and in an earlier publication for ${ }^{168} \mathrm{Yb}$ [ref. ${ }^{1}$ )], namely that the deformations of both ground-state and shape isomeric minima found with the expectation-value method agree closely with the results obtained self-consistently. This, of course, is closely connected to our conclusion that the shell effects are mainly determined by the shape given to the average potential and therefore can be approximately reproduced in our method without the requirement of self-consistency.

\section{Illustrations of calculational details}

\subsection{REOCCUPATION}

The effects of reoccupation on the total energy of ${ }^{240} \mathrm{Pu}$ are studied in the case of interaction SKY X. A schematic diagram illustrating the idea of reoccupation described in subsect. 2.2 is shown in fig. 13. The deformation energy curves of ${ }^{240} \mathrm{Pu}$ along $h=\alpha=0$ arc given in fig. 14 to illustrate the effects of reoccupation with or without scaling.

\subsection{PAIRING}

The effects of pairing on the deformation energy of ${ }^{240} \mathrm{Pu}$ are shown in fig. 15 for the case of the interaction SKY III. It is seen that its effect on the magnitude of the deformation energy is not very large, even in the case of the surface-dependent pairing which has strength proportional to the surface area of the nucleus. However, pairing effects smooth out the deformation energy curve obtained without pairing. This is similar to what one observed in the Strutinsky-type calculations.

\subsection{SCALING}

The effects of scaling have been shown already in fig. 14 for the interaction SKY X. It is important in bringing down the total energy of ${ }^{240} \mathrm{Pu}$ calculated by the expectation-value method which always gives binding energies less than the self-consistent results; and it is more appreciable in large deformations than in small deformations. The scale factors in the case of other interactions are shown in fig. 16 where one observes that they are always about $1 \%$ around unity.

\subsection{VARIATION OF POTENTIAL PARAMETERS}

As stated in subsect. 2.1, our results approach those of the self-consistent calculations if the energies are minimized with respect to all the parameters in the deformed WoodsSaxon potential. Since this is not feasible to be carried out in practice, we only give 


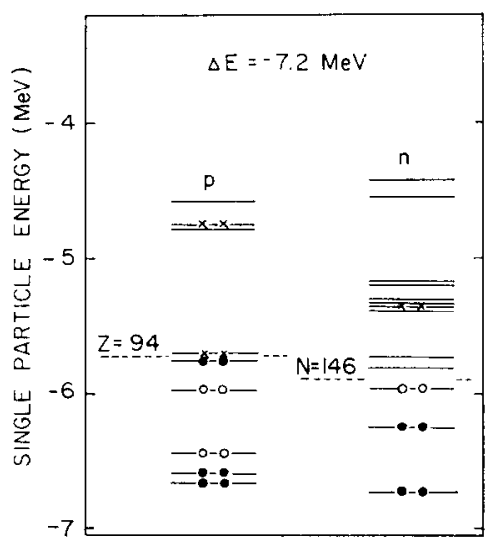

Fig. 13. Single-particle states $e_{t}$ of the phenomenological Woods-Saxon potential for ${ }^{240} \mathrm{Pu}$, taken at the deformation $c=1.51(h=\alpha=0)$. The figure shows the position of the Fermi energy for the protons and the neutrons by the broken line, and the optimal occupation for SKY X according to eq. (2.23); the latter is indicated as particle-hole excitations in the spectrum of the $e_{i}$. The decrease in the total binding energy in this case amounts to $-7.2 \mathrm{MeV}$, and is one of the largest so far met.

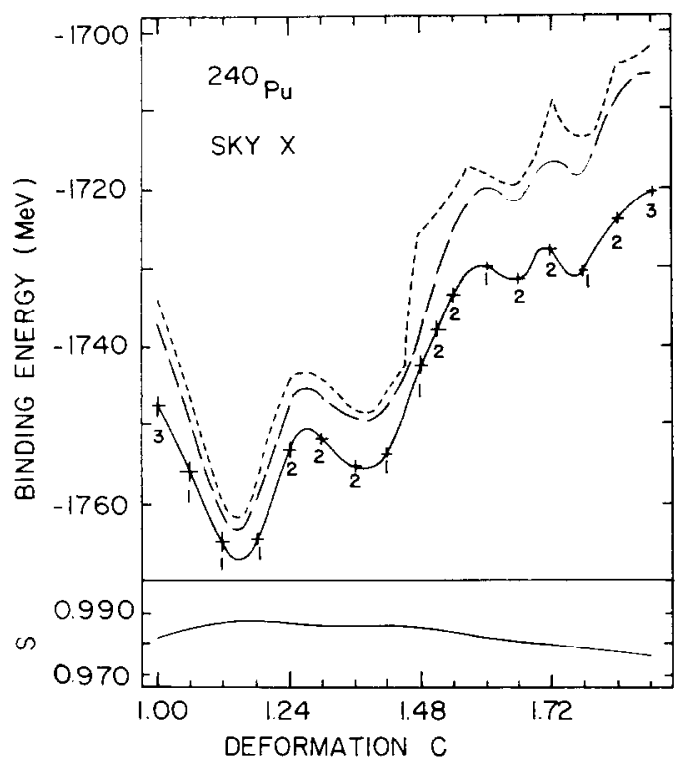

Fig. 14. The effect of reoccupation. Upper part: Deformation energy curves of ${ }^{240} \mathrm{Pu}$ along $h=\alpha=0$ in the $\{c, h, \alpha\}$ space, calculated by the expectation-value method with SKY X and Woods-Saxon single-particle wave functions, the latter are obtained using cut-offs $N_{E}-12$ for protons and $N_{E}=13$ for neutrons. The first curve (dotted line) is obtained with occupations taken according to the lowest Woods-Saxon single-particle energies $e_{i}$; the second curve (dashed line) is obtained with the optimal occupations for the interaction SKY X, evaluated according to eq.(2.23); and the lower curve (solid line) is the same as the second but with scale corrected according to eq. (2.22). The numbers on the lower curve show how many levels have been reoccupied. Lower part: The scaling factor $s$, defined by eq. (2.21), is plotted versus the deformation $c$. As the figure shows, the mean variation is less than $1 \%$, indicating that the "volume conservation" chosen for the phenomenological potential is rather good. 
some examples in the case of ${ }^{208} \mathrm{~Pb}$ at the spherical shape. They are shown in fig. 17. Since the parameters used have been adjusted before to fit, to some extent, the experimental single-particle levels of ${ }^{208} \mathrm{~Pb}$, the variational calculations improve only slightly the total binding energy. What we want to emphasize is that the total energy

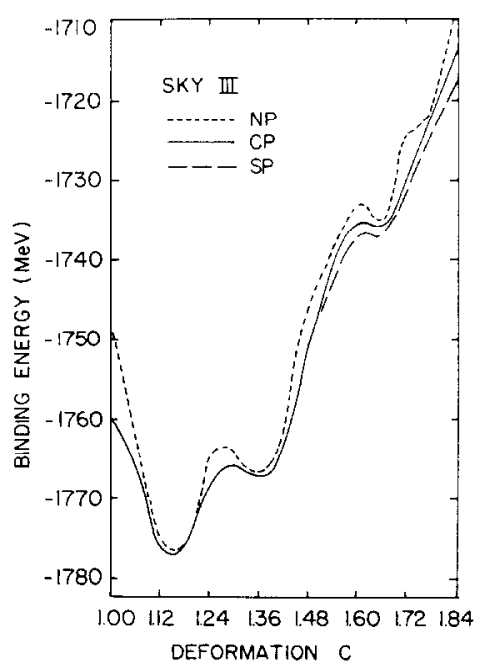

Fig. 15. The effect of pairing. Deformation energy of ${ }^{24}{ }^{\circ} \mathrm{Pu}$ along $h=\alpha=0$, calculated by the expectation-value method with SKY III and the Woods-Saxon single-particle wave functions. Line NP no pairing is included and the occupation is according to the lowest Woods-Saxon single-particle energies $e_{i}$. Line CP pairing with constant $\tilde{\Delta}$ is used. Line SP surface pairing is used, i.e., $G_{\mathrm{n}}, G_{\mathrm{p}} \propto$ surface area. Scaling is not included in all cases.

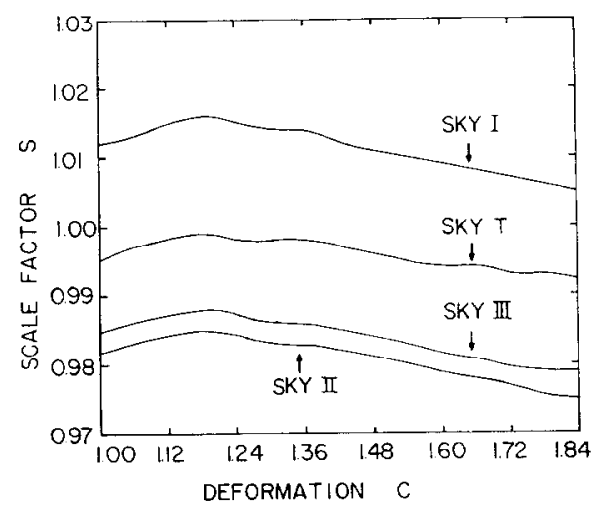

Fig. 16. Scale factors found for the deformation energy curves of ${ }^{240} \mathrm{Pu}$ along $h=\alpha=0$. The curves are evaluated with constant pairing.

calculated by the expectation-value method depends very much on the potential parameters used, as seen in the figure. Although our parameters are close to the spherical ones for ${ }^{208} \mathrm{~Pb}$, they may not be good enough for ${ }^{240} \mathrm{Pu}$. This seems to be suggested by the large fission barriers we have obtained previously. 

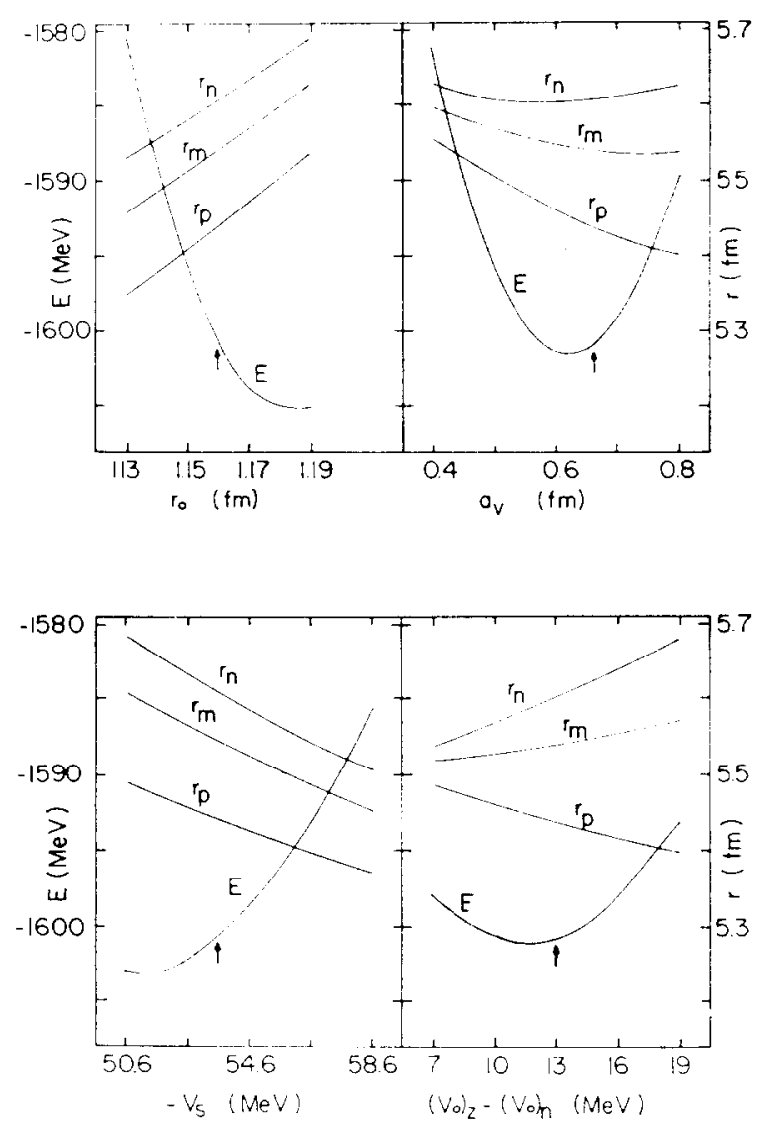

Fig. 17. Variations of potential parameters. The energy expectation value $E$ and the rms radii $r_{\mathrm{n}}$, $r_{p}$ and $r_{m}$ for neutron, proton and the nucleus, respectively, are shown as functions of some of the potential parameters: the radius constant $r_{0}$ (upper left), the potential diffusivity $a_{\mathrm{v}}$ (upper right), the isoscalar part of the potential depth $V_{s}$ (lower left) and the difference between the isovector part of the nuclear potential depth for the neutron $\left(V_{0}\right)_{N}$ and the proton $\left(V_{0}\right)_{Z}$ (lower right). Cut-off parameters $\left(N_{E}, N_{0}\right)$ are $(12,20)$ and $(13,20)$ for protons and neutrons, respectively. The standard values of these potential parameters are indicated by arrows. Note that the present values are rather close to the saturation values, but that the total energy could be improved to some extent by a better parameter set. The above results have not becn scale corrected.

\subsection{CONTRIBUTIONS TO THE TOTAL ENERGY}

The contributions to the total energy from various terms of the Skyrme interaction are shown in fig. 18 for the interaction SKY III. The Coulomb energy as a function of deformation is shown in fig. 19 along with that calculated in the liquid-drop model. The dominant term in the Skyrme interaction is $E_{0}$ which has a similar shell structure as a Strutinsky curve. All the other terms have relatively small contributions compared to $E_{0}$, but their shell structures are apparently seen to be significant. The 


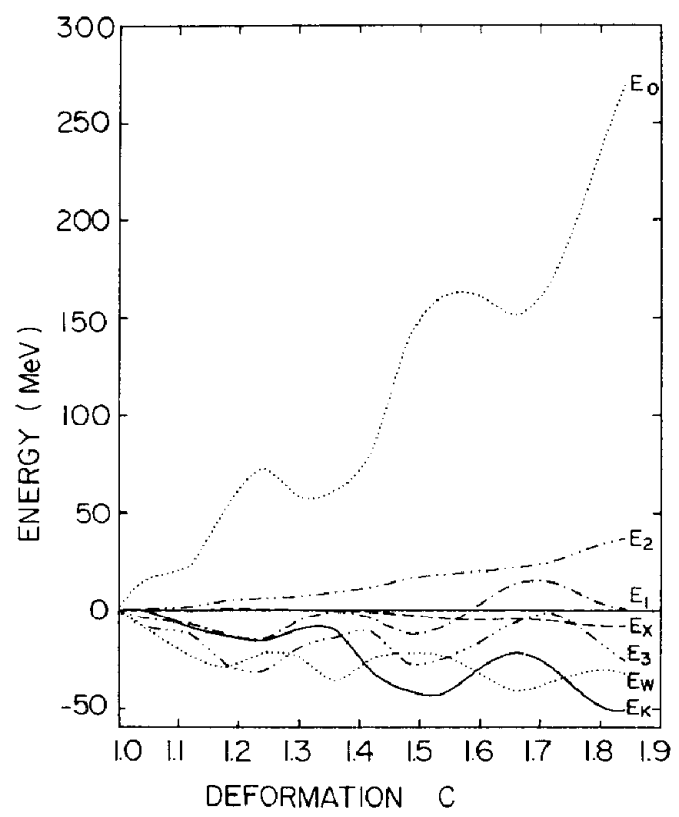

Fig. 18. Contributions of various parts of the total Skyrme Hamiltonian to the deformation energy of ${ }^{240} \mathrm{Pu}$. Calculations are made with SKY III using the expectation-value method. The constant pairing is included but not scaling. At spherical shape, the actual values in $\mathrm{MeV}$ of each term are $4558.0,-12121.3,145.8,2224.4,-766.8,-73.0$ and 3319.1 for $E_{\mathrm{k}}, E_{0}, E_{\mathrm{x}}, E_{1}, E_{2}, E_{w}$ and $E_{3}$, respectively.

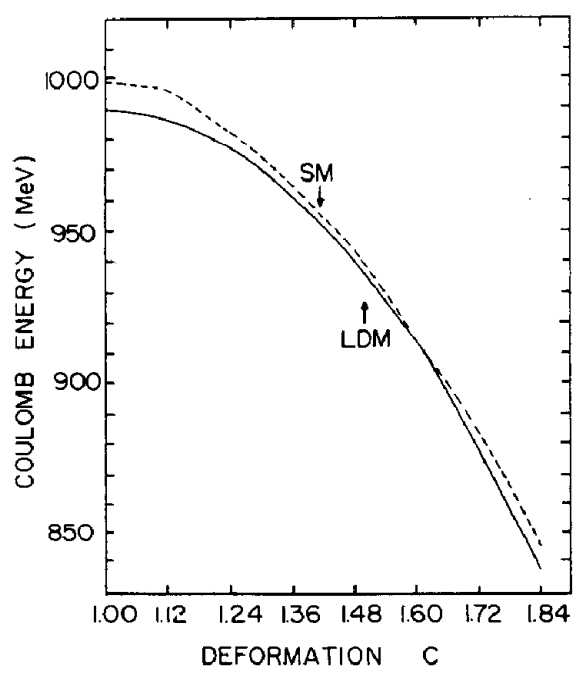

Fig. 19. Coulomb energy of ${ }^{240} \mathrm{Pu}$ versus deformation $c(h=\alpha=0)$. Results obtained in the expectation-value method (SM) include the constant pairing and the exchange Coulomb energy in the Slater approximation. The solid curve shows the Coulomb energy found within the liquid-drop model. 


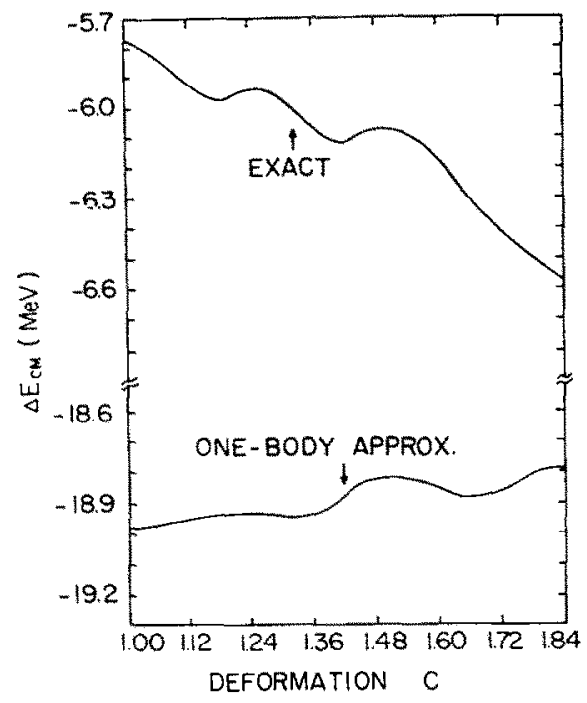

Fig. 20. Center-of-mass kinetic energy correction $\Delta E_{\text {c.an.. }}$, eq. (2.27), for ${ }^{240} \mathrm{Pu}$ along $h=\alpha=0$. The lower curve corresponds to the one-body approximation, which overestimates the effect by a factor of 3. The upper curve is calculated exactly. Note the little variation of both curves with deformation.

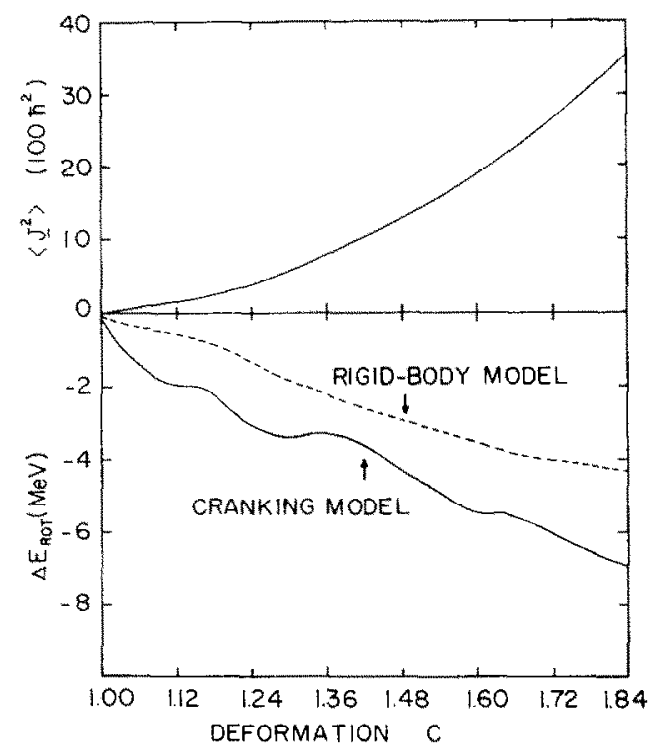

Fig. 21. Upper part: Average value of the total angular momentum squared $\left\langle J^{2}\right\rangle$ in ${ }^{240} \mathrm{Pu}$ along $h=\alpha=0$. Lower part: Rotational energy correction $\Delta E_{\mathrm{ret}}$ eq. (2.28), as a function of deformation $c$. The dashed curve is obtained with the rigid-body value of the moment of inertia $\mathscr{A}_{\mathrm{RB}}=m A$ $\left(\left\langle z^{2}\right\rangle+\frac{1}{2}\left\langle p^{2}\right\rangle\right)$; in the solid curve, the cranking model is used for the moment of inertia. The constant pairing is included in both cases. 
Coulomb energy calculated with the expectation-value method is rather smooth and is very close to that obtained in the liquid-drop model.

\subsection{CORRECTIONS TO THE TOTAL ENERGY}

The corrections due to the c.m. motion and the rotational motion are shown in figs. 20 and 21 respectively. The effect of the c.m. correction on the deformation energy is small, but that of the rotational correction is appreciable. This is in contrast to Strutinsky-type calculations, where only the fluctuating parts of these corrections contribute to the shell corrections ${ }^{26,27}$ ), which are therefore small even in the case of rotational corrections.

\section{Conclusions}

Motivated by the desire to relate the shell corrections to the two-body interactions, we have proposed the expectation-value method in calculating the binding and deformation energies of nuclei. Aside of self-consistency, this method is completely microscopic. The binding energies and deformation energy curves so calculated have been checked against self-consistent calculations and are seen to agree with the latter not only qualitatively but often quantitatively. The importance of self-consistency shows most clearly in the height of fission barriers.

Since the expectation-value method is computationally much faster than the constrained HF method, we have been able to construct two-dimensional energy surfaces without much effort. When compared to those obtained with the well-established Strutinsky method, they are seen to have almost identical shell-structures, although the absolute deformation energies do not agree quantitatively at large deformations.

Improvements on the expectation-value method can be pursued in the following directions,

(i) One can readjust the Skyrme parameters as we did in SKY T such that the requirement of self-consistency is almost met when the wave functions from the deformed Woods-Saxon potential are used.

(ii) One can perform some variational calculations with respect to the potential parameters to approach the self-consistent calculations sytematically.

(iii) One can extract the smoothed HF potential from the existing Skyrme interaction and then fit the parameters of the phenomenological potential directly to this smonthed potential.

The last approach is particularly interesting and useful, as the difference between the energy calculated with the HF method and that calculated with the expectationvalue method, using single-particle wave functions obtained from the smoothed $\mathrm{HF}$ field, is of the second order of the difference between the two corresponding densities. As shown recently by Brack and Quentin ${ }^{28}$ ), this difference is small. The third approach will thus provide a very good approximation to the self-consistent calculations and is still computationally fast. 
We acknowledge Drs. Negele and Vautherin for making their DME and CHF codes available to us.

\section{Appendix}

In this appendix, we give the explicit expressions for the various density functions needed in the evaluation of the nuclear binding energy.

The various single-particle density functions are defined by the following expressions

$$
\begin{gathered}
\rho_{i}(\boldsymbol{r})=n_{i} \psi_{i}^{*}(\boldsymbol{r}) \psi_{i}(\boldsymbol{r}), \\
\chi_{i}(\boldsymbol{r})=n_{i} \psi_{i}^{*}(\boldsymbol{r}) \nabla^{2} \psi_{i}(\boldsymbol{r}), \\
\tau_{i}(\boldsymbol{r})=n_{i} \nabla \psi_{i}^{*}(\boldsymbol{r}) \cdot \nabla \psi_{i}(\boldsymbol{r}), \\
\pi_{i}(\boldsymbol{r})=n_{i}(-i) \sum_{s, s^{\prime}} \nabla \phi_{i}^{*}(\boldsymbol{r}, s) \cdot \nabla \phi_{i}\left(\boldsymbol{r}, s^{\prime}\right) \times\left(s|\boldsymbol{\sigma}| s^{\prime}\right) .
\end{gathered}
$$

In the above, $n_{i}$ is the occupation number of the single-particle state $|i\rangle$, which has wave function

$$
\psi_{i}(\boldsymbol{r})=\sum_{s} \phi_{i}(\boldsymbol{r}, s) \chi_{s}
$$

where $\chi_{s}$ and $\phi_{i}(r, s)$ are the spin and the spatial part respectively. The density function $\chi_{i}(r)$ is useful in evaluating $\nabla^{2} \rho_{i}(r)$ as the latter can be expressed as

$$
\nabla^{2} \rho_{i}(\boldsymbol{r})=2\left(\tau_{i}(\boldsymbol{r})+\chi_{i}(\boldsymbol{r})\right)
$$

Since the Woods-Saxon potential is assumed to be axially symmetric, the singleparticle states are labeled by the $z$-component $K$ of the angular momentum. We express $\phi_{i}(\boldsymbol{r}, s)$ in terms of the cylindrical harmonic-oscillator wave functions

$$
\phi_{i}(\boldsymbol{r}, s)=\sum_{\alpha_{s}} C_{i}^{\alpha_{s}} \phi_{\alpha_{s}}(r) .
$$

The harmonic-oscillator functions $\phi_{\alpha_{s}}(\boldsymbol{r})$ have the following form

where

$$
\phi_{\alpha_{s}}(r)=\phi_{n_{z}}(z) \phi_{n_{\rho}}^{\Lambda_{s}}(\rho) \phi_{A_{s}}(\varphi),
$$

$$
\begin{gathered}
\phi_{n_{z}}(z)=\left(m \omega_{z} / \hbar\right)^{\frac{1}{4}} \exp \left(-\frac{1}{2} \xi^{2}\right) h_{n_{z}}(\xi), \\
\phi_{n_{\rho}}^{\Lambda_{s}}(\rho)=\left(2 m \omega_{\rho} / \hbar\right)^{\frac{1}{2}} \exp \left(-\frac{1}{2} \zeta\right) g_{n_{\rho}}^{\Lambda_{s}}(\zeta), \\
\phi_{\Lambda_{s}}(\varphi)=(2 \pi)^{-\frac{1}{2}} \exp \left(i \Lambda_{s} \varphi\right),
\end{gathered}
$$

with

$$
\begin{gathered}
\Lambda_{s}=K_{i}-s, \\
\xi=\left(m \omega_{z} / \hbar\right)^{\frac{1}{2}} z, \quad \zeta^{\frac{1}{2}}=\left(m \omega_{\rho} / \hbar\right)^{\frac{1}{2}} \rho .
\end{gathered}
$$

The functions $h_{n_{z}}(\xi)$ and $g_{n_{\rho}}^{\Lambda_{s}}(\zeta)$ are the modified Hermite and Laguerre polynomials

$$
\begin{gathered}
h_{n_{z}}(\xi)=\left(2^{n_{z}} n_{z} ! \pi^{\frac{1}{2}}\right)^{-\frac{1}{2}} H_{n_{z}}(\xi), \\
g_{n_{\rho}}^{\Lambda_{s}}(\zeta)=\left(n_{\rho} !\left(n_{\rho}+\Lambda_{s}\right) !\right)^{\frac{1}{2}} \zeta^{\frac{1}{2} A_{s}} L_{n_{\rho}}^{A_{s}}(\zeta),
\end{gathered}
$$


where the usual Hermite and Laguerre polynomials are denoted by $H_{n_{z}}(\xi)$ and $L_{n_{\rho}}^{A_{s}}(\zeta)$.

We introduce the following definitions

$$
\begin{gathered}
\tilde{h}_{n_{z}}(\xi)=\mathrm{e}^{\frac{1}{\xi^{2}}} \frac{\mathrm{d}}{\mathrm{d} \xi}\left[\mathrm{e}^{-\frac{1}{2} \xi^{2}} h_{n_{z}}(\xi)\right], \\
\tilde{g}_{n_{\rho}}^{A_{s}}(\zeta)=2 \zeta^{\frac{1}{2}} \mathrm{e}^{\frac{1}{2} \zeta} \frac{\mathrm{d}}{\mathrm{d} \zeta}\left[\mathrm{e}^{-\frac{1}{2} \zeta} g_{n_{\rho}}^{\Lambda_{s}}(\zeta)\right], \\
F_{\alpha_{s}}^{(1)}(\xi, \zeta)=h_{n_{z}}(\xi) g_{n_{\rho}}^{A_{s}}(\zeta), \\
F_{\alpha_{s}}^{(2)}(\xi, \zeta)=h_{n_{z}}(\zeta) g_{n_{\rho}}^{\Lambda_{s}}(\zeta)\left[a_{z}\left(\xi^{2}-2 n_{z}-1\right)+a_{\rho}\left(\zeta-2 n_{\perp}-2\right)\right] l^{2}, \\
F_{\alpha_{s}}^{(3)}(\xi, \zeta)=\tilde{h}_{n_{z}}(\xi) g_{n_{\rho}}^{A s}(\zeta) a_{z}^{\frac{1}{2}} l, \\
F_{\alpha_{s}}^{(4)}(\xi, \zeta)=h_{n_{z}}(\xi) \tilde{g}_{n_{\rho}}^{\Lambda_{s}}(\zeta) a_{\rho}^{\frac{1}{2}} l, \\
F_{\alpha_{s}}^{(5)}(\xi, \zeta)=h_{n_{z}}(\xi) g_{n_{\rho}}^{A s}(\zeta)\left(\Lambda_{s} / \zeta^{\frac{1}{2}}\right) a_{\rho}^{\frac{1}{2}} l,
\end{gathered}
$$

where

$$
\begin{gathered}
n_{\perp}=2 n_{\rho}+\Lambda_{s}, \quad l=\left(m \omega_{0} / \hbar\right)^{\frac{1}{2}}, \\
a_{z}=\omega_{z} / \omega_{0}, \quad a_{\rho}=\omega_{\rho} / \omega_{0}, \quad \omega_{0}^{3}=\omega_{\rho}^{2} \omega_{z} .
\end{gathered}
$$

Then we can write the Woods-Saxon wave functions and their derivatives in the following form

$$
\begin{gathered}
\psi_{i}(\boldsymbol{r})=N \mathrm{e}^{-\frac{1}{2} \xi^{2}} \mathrm{e}^{-\frac{1}{2} \zeta} \sum_{s} A_{i s}^{(1)}(\xi, \zeta) \mathrm{e}^{i \Lambda_{s}} \chi_{s}, \\
\nabla^{2} \psi_{i}(\boldsymbol{r})=N \mathrm{e}^{-\frac{1}{2} \xi^{2}} \mathrm{e}^{-\frac{1}{2} \zeta} \sum_{s} A_{i s}^{(2)}(\zeta, \zeta) \mathrm{e}^{i \Lambda_{s}} \chi_{s}, \\
\frac{\partial}{\partial z} \psi_{i}(\boldsymbol{r})=N \mathrm{e}^{-\frac{1}{2} \xi^{2}} \mathrm{e}^{-\frac{1}{\zeta} \zeta} \sum_{s} A_{i s}^{(3)}(\xi, \zeta) \mathrm{e}^{i \Lambda_{s}} \chi_{s}, \\
\frac{\partial}{\partial \rho} \psi_{i}(\boldsymbol{r})=N \mathrm{e}^{-\frac{1}{2} \xi^{2}} \mathrm{e}^{-\frac{1}{2} \zeta} \sum_{s} A_{i s}^{(4)}(\xi, \zeta) \mathrm{e}^{i \Lambda_{s}} \chi_{s}, \\
-\frac{i}{\rho} \frac{\partial}{\partial \varphi} \psi_{i}(\boldsymbol{r})=N \mathrm{e}^{-\frac{1}{2} \xi^{2}} \mathrm{e}^{-\frac{1}{2} \zeta} \sum_{s} A_{i s}^{(5)}(\zeta, \zeta) \mathrm{e}^{i \Lambda_{s}} \chi_{s},
\end{gathered}
$$

where

$$
\begin{aligned}
A_{i s}^{(n)}(\xi, \zeta) & =\sum_{\alpha_{s}} C_{i}^{\alpha_{s}} F_{\alpha_{s}}^{(n)}(\xi, \zeta) \\
N & =l^{\frac{3}{2}} \pi^{-\frac{1}{2}}
\end{aligned}
$$

With the above expressions, the single-particle density functions are given by

$$
\begin{aligned}
\rho_{i}(\xi, \zeta) & =N^{2} \mathrm{e}^{-\xi^{2}} \mathrm{e}^{-\zeta} D_{\rho_{i}}(\xi, \zeta), \\
\tau_{i}(\xi, \zeta) & =N^{2} \mathrm{e}^{-\xi^{2}} \mathrm{e}^{-\zeta} D_{\tau_{i}}(\xi, \zeta), \\
\chi_{i}(\xi, \zeta) & =N^{2} \mathrm{e}^{-\xi^{2}} \mathrm{e}^{-\zeta} D_{\chi_{i}}(\xi, \zeta), \\
\pi_{i}(\xi, \zeta) & =N^{2} \mathrm{e}^{-\xi^{2}} \mathrm{e}^{-\zeta} D_{\pi_{i}}(\xi, \zeta),
\end{aligned}
$$


where

$$
\begin{gathered}
D_{\rho_{i}}(\xi, \zeta)=n_{i}\left\{\left[A_{i+}^{(1)}\right]^{2}+\left[A_{i-}^{(1)}\right]^{2}\right\}, \\
D_{\chi_{i}}(\xi, \zeta)=n_{i}\left\{A_{i+}^{(1)} A_{i+}^{(2)}+A_{i-}^{(1)} A_{i-}^{(2)}\right\} \\
D_{\tau_{i}}(\xi, \zeta)=n_{i}\left\{\left[A_{i+}^{(3)}\right]^{2}+\left[A_{i-}^{(3)}\right]^{2}+\left[A_{i+}^{(4)}\right]^{2}+\left[A_{i-}^{(4)}\right]^{2}+\left[A_{i+}^{(5)}\right]^{2}+\left[A_{i-}^{(5)}\right]^{2}\right\}, \\
D_{\pi_{i}}(\zeta, \zeta)=2 n_{i}\left\{\left[A_{i+}^{(4)} A_{i-}^{(5)}-A_{i-}^{(4)} A_{i+}^{(5)}\right]\right. \\
\left.+A_{i-}^{(3)}\left(A_{i+}^{(4)}-A_{i+}^{(5)}\right)-A_{i+}^{(3)}\left(A_{i-}^{(4)}+A_{i-}^{(5)}\right)\right\} .
\end{gathered}
$$

\section{References}

1) C. M. Ko, H. C. Pauli, M. Brack and G. E. Brown, Phys. Lett. 45B (1973) 433

2) C. M. Ko, Ph.D. thesis, SUNY at Stony Brook, 1973

3) V. M. Strutinsky, Nucl. Phys. A95 (1967) 420; A122 (1968) 1

4) M. Brack, J. Damgaard, A. S. Jensen, H. C. Pauli, V. M. Strutinsky and C. Y. Wong, Rev. Mod. Phys. 44 (1972) 320

5) J. R. Nix, Ann. Rev. Nucl. Sci. 22 (1972) 65

6) H. C. Pauli, Phys. Rep. 7C (1973) 36

7) T. H. R. Skyrme, Nucl. Phys. 9 (1959) 615

8) D. Vautherin and D. M. Brink, Phys. Rev. C5 (1972) 626

9) J. W. Negele and D. Vautherin, Phys. Rev. C5 (1972) 1472

10) D. Vautherin, Phys. Rev. C7 (1973) 296

11) H. Flocard, P. Quentin, A. K. Kerman and D. Vautherin, Nucl. Phys. A203 (1973) 433

12) H. Flocard et al., Proc. 3rd Int. Symp. on chemistry and physics of fission, IAEA Vienna, 1974 , p. 221

13) D. G. Ravenhall, C. D. Bennett and C. J. Pethick, Phys. Rev. Lett. 28 (1972) 978

14) M. Brack and P. Quentin, Proc. Int. Workshop II on gross properties of nuclei and nuclear excitations, Hirshegg, Austria, 1974 (AED-Conf-74-025-000) p. 14

15) S. A. Moszkowski, Phys. Rev. C2 (1970) 482

16) J. W. Ehlers and S. A. Moszkowski, Phys. Rev. C6 (1972) 217

17) K. R. Lassey and A. B. Volkov, Phys. Lett. 36B (1971) 4

18) K. R. Lassey, Nucl. Phys. A192 (1972) 177

19) W. H. Bassichis, A. K. Kerman, C. F. Tsang, D. R. Tuerpe and L. Wilets, in Magic without magic: Archibald Wheeler (Freeman, San Francisco, 1972)

20) W. H. Bassichis, C. F. Tsang, D. R. Tuerpe and L. Wilets, Phys. Rev. Lett. 30 (1973) 294

21) M. Brack, T. Ledergerber, H. C. Pauli and A. S. Jensen, Nucl. Phys. A234 (1974) 185

22) J. Damgaard, H. C. Pauli, V. V. Pashkevich and V. M. Strutinsky, Nucl. Phys. A135 (1969) 432

23) W. D. Myers, Nucl. Phys. A145 (1970) 387

24) W. D. Myers and W. J. Swiatecki, Nucl. Phys. 81 (1966) 1; Ann. of Phys. 55 (1969) 395

25) H. C. Pauli, C. M. Ko, M. Brack and G. E. Brown, Proc. Int. Conf. on nuclear physics, Munich, vol. 1 (1973) p. 586

26) I. Kelson and Y. Shoshani. Phys. Lett. 40B (1972) 58

27) C. K. Ross and C. S. Warke, Phys. Rev. Lett. 30 (1973) 55

28) M. Brack and P. Quentin, Proc. 3rd Int. Symp. on chemistry and physics of fission, IAEA Vienna, 1974, p. 231

29) H. C. Pauli and T. Ledergerber, Nucl. Phys. A175 (1971) 545 